\title{
SQUARE-MEAN PSEUDO ALMOST PERIODIC SOLUTIONS OF CLASS $r$ UNDER THE LIGHT OF MEASURE THEORY
}

\author{
MOHAMADO KIEMA AND ISSA ZABSONRE*
}

\begin{abstract}
The aim of this work is to present new concept of square-mean pseudo almost periodic of class $r$ using the measure theory. We use the $(\mu, \nu)$-ergodic process to define the spaces of $(\mu, \nu)$-pseudo almost periodic processes of class $r$ in the square-mean sense. We present many interesting results on those spaces like completeness and composition theorems and we study the existence and the uniqueness of the square-mean $(\mu, \nu)$-pseudo almost periodic solutions of class $r$ for the stochastic evolution equation.
\end{abstract}

\section{INTRODUCTION}

In this work, we study some properties of the square-mean $(\mu, \nu)$-pseudo almost periodic process using the measure theory and we used those results to study the following stochastic evolution equations in a Hilbert space $H$,

$$
d x(t)=\left[A x(t)+L\left(x_{t}\right)+f(t)\right] d t+g(t) d W(t),
$$

where $A: D(A) \subset H$ is the infinitesimal generator of a $C_{0}$-semigroup $(T(t))_{t \geq 0}$ on $H$, $f, g: \mathbb{R} \rightarrow L^{2}(P, H)$ are two stochastic processes and $W(t)$ is a two-sided and standard one-dimensional Brownian notion defined on the filtered probability space $\left(\Omega, \mathcal{F}, P, \mathcal{F}_{t}\right)$ with $\mathcal{F}_{t}=\sigma\{W(u)-W(v) \mid u, v \leq t\}$ and $L$ is a bounded linear operator from $C$ into $L^{2}(P, H)$. $C=C\left([-r, 0] ; L^{2}(P, H)\right)$ denotes the space of continuous functions from $[-r, 0]$ to $L^{2}(P, H)$ endowed with the uniform topology norm. For every $t \geq 0, x_{t}$ denotes the history function of $C$ defined by $x_{t}(\theta)=x(t+\theta)$ for $-r \leq \theta \leq 0$.

We assume $(H,\|\|$,$) is a real separable Hilbert space and L^{2}(P, H)$ is the space of all $H$-valued random variables $x$ such that

$$
\mathbb{E}\|x\|^{2}=\int_{\Omega}\|x\|^{2} d P<+\infty .
$$

This work is an extension of [14] whose authors have studied equation (1.1) in the deterministic case. Some recent contributions concerning square-mean pseudo almost periodic solutions for abstract differrential equations similar to equation (1.1) have been made. For example in [10] the authors studied equation(1.1) without the operator $L$. They showed that the equation has a unique square-mean $\mu$-pseudo almost periodic mild solution on $\mathbb{R}$ when $f$ and $g$ are square

Université Joseph KI-ZERBO, B.P.7021 Ouagadougou 03, Burkina Faso

*CORRESPONDING AUTHOR

E-mail addresses: hamadkiema@gmail.com, zabsonreissa@yahoo.fr.

Key words and phrases. measure theory; ergodicity; $(\mu, \nu)$-pseudo almost periodic function; evolution equations; partial functional differential equations; Stochastic processes; stochastic evolution equations.

Received 06/11/2021. 
mean pseudo almost periodic functions.

In [5] the authors studied the square-mean almost periodic solutions to a class of nonautonomous stochastic differential equations without our operator $L$ and without delay on a separable real Hilbert space. They established the existence and uniqueness of a square-mean almost periodic mild solution to those nonautonomous stochastic differential equations with the 'AcquistapaceTerreni' conditions.

In [9] The authors established the existence, uniqueness and stability of square-mean $\mu$-pseudo almost periodic (resp. automorphic) mild solution to a linear and semilinear case of the stochastic evolution equations in case when the functions forcing are both continuous and $S^{2}-\mu$-pseudo almost periodic (resp. automorphic) and verify some suitable assumptions.

This work is organized as follow, in section 2, we give spectral decomposition of phase space in section 3 we study square-mean $(\mu, \nu)$-ergodic process of class $\mathrm{r}$, in section 4 we study squaremean $(\mu, \nu)$-pseudo almost process functions and properties and last section is devoted to an application.

\section{Spectral Decomposition}

To equation (1.1), associate the following initial value problem

$$
\left\{\begin{array}{l}
d u_{t}=\left[A u_{t}+L\left(u_{t}\right)+f(t)\right] d t+g(t) d W(t) \text { for } t \geq 0 \\
u_{0}=\varphi \in C=C\left([-r, 0], L^{2}(P, H)\right)
\end{array}\right.
$$

where $f: \mathbb{R}^{+} \rightarrow L^{2}(P, H)$ and $g: \mathbb{R}^{+} \rightarrow L^{2}(P, H)$ are stochastic processes continuous.

Definition 2.1. We say that a continuous function $u$ from $\left[-r,+\infty\left[\right.\right.$ into $L^{2}(P, H)$ is an integral solution of equation, if the following conditions hold:

(1) $\int_{0}^{t} u(s) d s \in D(A)$ for $t \geq 0$,

(2) $u(t)=\phi(0)+A \int_{0}^{t} u(s) d s+\int_{0}^{t}\left(L\left(u_{s}\right)+f(s)\right) d s+\int_{0}^{t} g(s) d W(s)$, for $t \geq 0$,

(3) $u_{0}=\phi$.

If $\overline{D(A)}=L^{2}(P, H)$, the integral solution coincide with the known mild solutions. One can see that if $u(t)$ is an integral of equation 2.1, then $u(t) \in \overline{D(A)}$ for all $t \geq 0$, in particular $\phi(0) \in \overline{D(A)}$

Let us introduce the part $A_{0}$ of the operator $A$ in $\overline{D(A)}$ which defined by

$$
\left\{\begin{array}{l}
D\left(A_{0}\right)=\{x \in D(A): A x \in \overline{D(A)}\} \\
A_{0} x=A x \text { for } x \in D\left(A_{0}\right)
\end{array}\right.
$$

The following assumption is supposed:

$\left(\mathbf{H}_{\mathbf{0}}\right) A$ satisfies the Hille-Yosida condition.

Proposition 2.2. [2] $A_{0}$ generates a strongly continuous semigroup $\left(T_{0}(t)\right)_{t \geq 0}$ on $\overline{D(A)}$.

The phase space $\mathcal{C}_{0}$ of equation (2.1) is defined by

$$
C_{0}=\{\varphi \in C: \varphi(0) \in \overline{D(A)}\} .
$$


For each $t \geq 0$, the linear operator $\mathcal{U}(t)$ on $C_{0}$ is defined by

$$
\mathcal{U}(t)=v_{t}(., \varphi)
$$

where $v(., \varphi)$ is the solution of the following homogeneous equation

$$
\left\{\begin{array}{l}
\frac{d}{d t} v_{t}=A v_{t}+L\left(v_{t}\right) \text { for } t \geq 0 \\
v_{0}=\varphi \in C
\end{array}\right.
$$

Proposition 2.3. [3] $(\mathcal{U}(t))_{t \geq 0}$ is a strongly continuous semigroup of linear operators on $C_{0}$. Moreover, $(\mathcal{U}(t))_{t \geq 0}$ satisfies, for $t \geq 0$ and $\theta \in[-r, 0]$, the following translation property

$$
(\mathcal{U}(t) \varphi)(\theta)=\left\{\begin{array}{l}
(\mathcal{U}(t+\theta) \varphi)(0) \text { for } t+\theta \geq 0 \\
\varphi(t+\theta) \text { for } t+\theta \leq 0
\end{array}\right.
$$

Theorem 2.4. [3] Let $\mathcal{A}_{\mathcal{U}}$ defined on $C_{0}$ by

$$
\left\{\begin{array}{l}
D\left(\mathcal{A}_{\mathcal{U}}\right)=\left\{\varphi \in C^{1}([-r, 0] ; X) ; \varphi(0) \in \overline{D(A)} \text { and } \varphi^{\prime}(0)=A \varphi(0)+L(\varphi)\right\} \\
\mathcal{A}_{\mathcal{U}} \varphi=\varphi^{\prime} \text { for } \varphi \in D\left(\mathcal{A}_{\mathcal{U}}\right) .
\end{array}\right.
$$

Then $\mathcal{A}_{\mathcal{U}}$ is the infinitesimal generator of the semigroup $(\mathcal{U}(t))_{t \geq 0}$ on $C_{0}$.

Let $\left\langle X_{0}\right\rangle$ be the space defined by

$$
\left\langle X_{0}\right\rangle=\left\{X_{0} x: x \in X\right\}
$$

where the function $X_{0} x$ is defined by

$$
\left(X_{0} x\right)(\theta)=\left\{\begin{array}{l}
0 \text { if } \theta \in[-r, 0[, \\
x \text { if } \theta=0 .
\end{array}\right.
$$

The space $C_{0} \oplus\left\langle X_{0}\right\rangle$ equipped with the norm $\left|\phi+X_{0}\right|_{\mathcal{C}}=|\phi|_{\mathcal{C}}+|c|$ for $(\phi, c) \in C_{0} \times X$ is a Banach space and consider the extension $\mathcal{A}_{\mathcal{U}}$ defined on $C_{0} \oplus\left\langle X_{0}\right\rangle$ by

$$
\left\{\begin{array}{l}
D\left(\widetilde{\mathcal{A}_{\mathcal{U}}}\right)=\left\{\varphi \in C^{1}([-r, 0] ; X): \varphi \in D(A) \text { and } \varphi^{\prime} \in \overline{D(A)}\right\} \\
\widetilde{\mathcal{A}_{\mathcal{U}}} \varphi=\varphi^{\prime}+X_{0}\left(A \varphi+L(\varphi)-\varphi^{\prime}\right) .
\end{array}\right.
$$

Proposition 2.5. [3] Assume that $\left(\boldsymbol{H}_{\boldsymbol{O}}\right)$ holds. Then, $\widetilde{\mathcal{A}_{\mathcal{U}}}$ satisfies the Hille-Yosida condition on $C_{0} \oplus\left\langle X_{0}\right\rangle$ there exist $\widetilde{M} \geq 0, \widetilde{\omega} \in \mathbb{R}$ such that $] \widetilde{\omega},+\infty\left[\subset \rho\left(\widetilde{\mathcal{A}_{\mathcal{U}}}\right)\right.$ and

$$
\left|\left(\lambda I-\widetilde{\mathcal{A}_{\mathcal{U}}}\right)^{-n}\right| \leq \frac{\widetilde{M}}{(\lambda-\widetilde{\omega})^{n}} \text { for } n \in \mathbb{N} \text { and } \lambda>\widetilde{\omega}
$$

Moreover, the part of $\widetilde{\mathcal{A}_{\mathcal{U}}}$ on $D\left(\widetilde{\mathcal{A}_{\mathcal{U}}}\right)=C_{0}$ is exactly the operator $\widetilde{\mathcal{A}_{\mathcal{U}}}$.

Definition 2.6. The semigroup $(\mathcal{U}(t))_{t \geq 0}$ is hyperbolic if

$$
\sigma\left(\mathcal{A}_{\mathcal{U}}\right) \cap i \mathbb{R}=\emptyset
$$


For the sequel, we make the following assumption:

$\left(\mathbf{H}_{\mathbf{1}}\right) T_{0}(t)$ is compact on $\overline{D(A)}$ for every $t>0$.

Proposition 2.7. Assume that $\left(\boldsymbol{H}_{\boldsymbol{O}}\right)$ and $\left(\boldsymbol{H}_{\mathbf{1}}\right)$. then the semigroup $(\mathcal{U}(t))_{t \geq 0}$ is compact for $t>r$.

Proposition 2.8. Assume that $\left(\boldsymbol{H}_{\mathbf{1}}\right)$ holds. If the semigroup $(\mathcal{U}(t))_{t \geq 0}$ is hyperbolic then the space $C_{0}$ is decomposed as a direct sum

$$
C_{0}=S \oplus U
$$

of two $\mathcal{U}(t)$ invariant closed subspaces $S$ and $U$ such that the restricted semigroup on $\mathcal{U}$ is a group and there exist positive constant $\bar{M}$ and $\omega$ such that

$$
\begin{aligned}
& |\mathcal{U}(t) \varphi| \leq \bar{M} e^{-\omega t}|\varphi| \text { for } t \geq 0 \text { and } \varphi \in S \\
& |\mathcal{U}(t) \varphi| \leq \bar{M} e^{\omega t}|\varphi| \text { for } t \leq 0 \text { and } \varphi \in U,
\end{aligned}
$$

Where $S$ and $U$ are called respectively the stable and unstable space, $\Pi^{s}$ and $\Pi^{u}$ denote respectively the projection operator on $S$ and $U$.

\section{Square-Mean $(\mu, \nu)$-Ergodic Process of Class R}

Let $\mathcal{N}$ the Lebesgue $\sigma$-field of $\mathbb{R}$ and by $\mathcal{M}$ the set of all positive measures $\mu$ on $\mathcal{N}$ satisfying $\mu(\mathbb{R})=+\infty$ and $\mu([a, b])<\infty$, for all $a, b \in \mathbb{R}(a \leq b) . L^{2}(P, H)$ is a Hilbert space with following norm

$$
\|x\|_{L^{2}}=\left(\int_{\Omega}\|x\|^{2} d P\right)^{\frac{1}{2}}
$$

Definition 3.1. Let $x: \mathbb{R} \rightarrow L^{2}(P, H)$ be a stochastic process.

(1) $x$ said to be stochastically bounded if there exists $C>0$ such that

$$
\mathbb{E}\|x(t)\|^{2} \leq C \forall t \in \mathbb{R} .
$$

(2) $x$ is said to be stochastically continuous if

$$
\lim _{t \rightarrow s} \mathbb{E}\|x(t)-x(s)\|^{2}=0 \forall s \in \mathbb{R} .
$$

Denote by $S B C\left(\mathbb{R}, L^{2}(P, H)\right)$, the space of all stochastically bounded and continuous process. Otherwise, this space endowed the following norm

$$
\|x\|_{\infty}=\sup _{t \in \mathbb{R}}\left(\mathbb{E}\|x(t)\|^{2}\right)^{\frac{1}{2}}
$$

is a Banach space.

Definition 3.2. Let $\mu, \nu \in \mathcal{M}$. A stochastic process $f$ is said to be square-mean $(\mu, \nu)-$ ergodic if $f \in S B C\left(\mathbb{R}, L^{2}(P, H)\right)$ and satisfied

$$
\lim _{\tau \rightarrow+\infty} \frac{1}{\nu([-\tau, \tau])} \int_{-\tau}^{\tau} \mathbb{E}\|f(t)\|^{2} d \mu(t)=0 .
$$

We denote by $\mathcal{E}\left(\mathbb{R}, L^{2}(P, H), \mu, \nu\right)$, the space of all such process. 
Definition 3.3. Let $\mu, \nu \in \mathcal{M}$. A stochastic process $f$ is said to be square-mean $(\mu, \nu)-$ ergodic of class $r$ if $f \in S B C\left(\mathbb{R}, L^{2}(P, H)\right)$ and satisfied

$$
\lim _{\tau \rightarrow+\infty} \frac{1}{\nu([-\tau, \tau])} \int_{-\tau}^{\tau} \sup _{\theta \in[t-r, t]} \mathbb{E}\|f(\theta)\|^{2} d \mu(t)=0 .
$$

We denote by $\mathcal{E}\left(\mathbb{R}, L^{2}(P, H), \mu, \nu, r\right)$, the space of all such process.

For $\mu, \nu \in \mathcal{M}$ and $a \in \mathbb{R}$, we denote by $\mu_{a}$ the positive measure on $(\mathbb{R}, \mathcal{N})$ defined by

$$
\mu_{a}(A)=\mu(a+b: b \in A) \text { for } A \in \mathcal{N} .
$$

From $\mu, \nu \in \mathcal{M}$, we formulate the following hypothesis

$\left(\mathbf{H}_{2}\right)$ : For all $a \in \mathbb{R}$, there exists $\beta>0$ and a bounded intervall $I$ such that $\mu_{a}(A) \leq \beta \mu(A)$ when $A \in \mathcal{N}$ satisfies $A \cap I=\emptyset$.

$\left(\mathbf{H}_{3}\right)$ For all $a, b$ and $c \in \mathbb{R}$, such that $0 \leq a<b \leq c$, there exist $\delta_{0}$ and $\alpha_{0}>0$ such that

$$
|\delta| \geq \delta_{0} \Longrightarrow \mu(a+\delta, b+\delta) \geq \alpha_{0} \mu(\delta, c+\delta)
$$

$\left(\mathbf{H}_{4}\right)$ Let $\mu, \nu \in \mathcal{M}$ be such that $\limsup _{\tau \rightarrow+\infty} \frac{\mu([-\tau, \tau])}{\nu([-\tau, \tau])}=\alpha<\infty$.

Proposition 3.4. Assume that $\left(\boldsymbol{H}_{4}\right)$ holds. Then $\mathcal{E}\left(\mathbb{R}, L^{2}(P, H), \mu, \nu, r\right)$ is a Banach space with the norm $\|,\|_{\infty}$.

Proof. We can see that $\mathcal{E}\left(\mathbb{R} ; L^{2}(P, H), \mu, \nu, r\right)$ is a vector subspace of $\left.S B C\left(\mathbb{R}, L^{2}(P, H)\right)\right)$. To complete the proof, it is enough to prove that $\mathcal{E}\left(\mathbb{R} ; L^{2}(P, H), \mu, \nu, r\right)$ is closed in $S B C\left(\mathbb{R} ; L^{2}(P, H)\right)$. Let $\left(f_{n}\right)_{n}$ be a sequence in $\mathcal{E}\left(\mathbb{R} ; L^{2}(P, H), \mu, \nu, r\right)$ such that $\lim _{n \rightarrow+\infty} f_{n}=f$ uniformly in $S B C\left(\mathbb{R}, L^{2}(P, H)\right)$. From $\nu(\mathbb{R})=+\infty$, it follows $\nu([-\tau, \tau])>0$ for $\tau$ sufficiently large. Let $n_{0} \in \mathbb{N}$ such that for all $n \geq n_{0},\left\|f_{n}-f\right\|_{\infty}<\varepsilon$. Let $n \geq n_{0}$, then

$$
\begin{aligned}
\frac{1}{\nu([-\tau, \tau])} \int_{-\tau}^{+\tau}\left(\sup _{\theta \in[t-r, t]} \mathbb{E}\|f(\theta)\|^{2}\right) d \mu(t) \leq & \frac{2}{\nu([-\tau, \tau])} \int_{-\tau}^{+\tau}\left(\sup _{\theta \in[t-r, t]} \mathbb{E}\left\|f_{n}(\theta)-f(\theta)\right\|^{2}\right) d \mu(t) \\
& +\frac{2}{\nu([-\tau, \tau])} \int_{-\tau}^{+\tau}\left(\sup _{\theta \in[t-r, t]} \mathbb{E}\left\|f_{n}(\theta)\right\|^{2}\right) d \mu(t) \\
\leq & \frac{2}{\nu([-\tau, \tau])} \int_{-\tau}^{+\tau}\left(\sup _{t \in \mathbb{R}} \mathbb{E}\left\|f_{n}(t)-f(t)\right\|^{2}\right) d \mu(t) \\
& +\frac{2}{\nu([-\tau, \tau])} \int_{-\tau}^{+\tau}\left(\sup _{\theta \in[t-r, t]} \mathbb{E}\left\|f_{n}(\theta)\right\|^{2}\right) d \mu(t) \\
\leq & 2\left\|f_{n}-f\right\|_{\infty}^{2} \frac{\mu([-\tau, \tau])}{\nu([-\tau, \tau])} \\
& +\frac{2}{\nu([-\tau, \tau])} \int_{-\tau}^{+\tau}\left(\sup _{\theta \in[t-r, t]} \mathbb{E}\left\|f_{n}(\theta)\right\|\right) d \mu(t) .
\end{aligned}
$$

Consequently

$$
\limsup _{\tau \rightarrow+\infty} \frac{1}{\nu([-\tau, \tau])} \int_{-\tau}^{+\tau}\left(\sup _{\theta \in[t-r, t]} \mathbb{E}\|f(\theta)\|^{2}\right) d \mu(t) \leq 2 \alpha \varepsilon \text { for any } \varepsilon>0 .
$$

The following theorem is a characterization of square-mean $(\mu, \nu)$-ergodic processes (eventually $I=\emptyset$ ). 
Theorem 3.5. Assume that $\left(\boldsymbol{H}_{4}\right)$ holds and let $f \in S B C\left(\mathbb{R}, L^{2}(P, H)\right)$. Then the following assertions are equivalent:

i) $\mathcal{E}\left(\mathbb{R}, L^{2}(P, H), \mu, \nu, r\right)$
ii) $\lim _{\tau \rightarrow+\infty} \frac{1}{\nu([-\tau, \tau] \backslash I)} \int_{[-\tau, \tau] \backslash I} \sup _{\theta \in[t-r, t]} \mathbb{E}\|f(\theta)\|^{2} d \mu(t)=0$
iii) For any $\varepsilon>0, \lim _{\tau \rightarrow+\infty} \frac{\mu\left\{t \in[-\tau, \tau] \backslash I: \sup _{\theta \in[t-r, t]} \mathbb{E}\|f(\theta)\|^{2}>\varepsilon\right\}}{\nu([-\tau, \tau] \backslash I)}=0$

Proof. The proof is made like the proof of Theorem(2.13) in [6].

First, we show that $i$ ) is equivalent to $i i)$.

Denote by $A=\nu(I), B=\int_{I}\left(\sup _{\theta \in[t-r, t]} \mathbb{E}\|f(\theta)\|^{2}\right) d \mu(t)$. $A$ and $B$ belong to $\mathbb{R}$, since the interval $I$ is bounded and the process $f$ is stochastically bounded and continuous. For $\tau>0$ such that $I \subset[-\tau, \tau]$ and $\nu([-\tau, \tau] \backslash I)>0$, it follows

$$
\begin{aligned}
& \frac{1}{\nu([-\tau, \tau] \backslash I)} \int_{[-\tau, \tau] \backslash I}\left(\sup _{\theta \in[t-r, t]} \mathbb{E}\|f(\theta)\|^{2}\right) d \mu(t)=\frac{1}{\nu([-\tau, \tau])-A}\left[\int_{-\tau}^{\tau}\left(\sup _{\theta \in[t-r, t]} \mathbb{E}\|f(\theta)\|^{2}\right) d \mu(t)-B\right] \\
= & \frac{\nu([-\tau, \tau])}{\nu([-\tau, \tau])-A}\left[\frac{1}{\nu([-\tau, \tau])} \int_{[-\tau, \tau]}\left(\sup _{\theta \in[t-r, t]} \mathbb{E}\|f(\theta)\|^{2}\right) d \mu(t)-\frac{B}{\nu([-\tau, \tau])}\right] .
\end{aligned}
$$

From above equalities and the fact that $\nu(\mathbb{R})=+\infty$, ii) is equivalent to

$$
\lim _{\tau \rightarrow+\infty} \frac{1}{\nu([-\tau, \tau])} \int_{-\tau}^{+\tau}\left(\sup _{\theta \in[t-r, t]} \mathbb{E}\|f(\theta)\|^{2}\right) d \mu(t)=0
$$

that is i).

Now, we show that $i$ ii) implies $i i$ ).

Denote by $A_{\tau}^{\varepsilon}$ and $B_{\tau}^{\varepsilon}$ the following sets

$$
\begin{aligned}
& A_{\tau}^{\varepsilon}=\left\{t \in[-\tau, \tau] \backslash I: \sup _{\theta \in[t-r, t]} \mathbb{E}\|f(\theta)\|^{2}>\varepsilon\right\} \\
& \left.B_{\tau}^{\varepsilon}=\{t \in[-\tau, \tau] \backslash I): \sup _{\theta \in[t-r, t]} \mathbb{E}\|f(\theta)\|^{2} \leq \varepsilon\right\} .
\end{aligned}
$$

Assume that $i i i)$ holds, that is

$$
\lim _{\tau \rightarrow+\infty} \frac{\mu\left(A_{\tau}^{\varepsilon}\right)}{\nu([-\tau, \tau] \backslash I)}=0
$$

From the equality

$$
\begin{aligned}
\int_{[-\tau, \tau] \backslash I}\left(\sup _{\theta \in[t-r, t]} \mathbb{E}\|f(\theta)\|^{2}\right) d \mu(t)= & \int_{A_{\tau}^{\varepsilon}}\left(\sup _{\theta \in[t-r, t]} \mathbb{E}\|f(\theta)\|^{2}\right) d \mu(t) \\
& +\int_{B_{\tau}^{\varepsilon}}\left(\sup _{\theta \in[t-r, t]} \mathbb{E}\|f(\theta)\|^{2}\right) d \mu(t),
\end{aligned}
$$

then for $\tau$ sufficiently large

$$
\frac{1}{\nu([-\tau, \tau] \backslash I)} \int_{[-\tau, \tau] \backslash I}\left(\sup _{\theta \in[t-r, t]} \mathbb{E}\|f(\theta)\|^{2}\right) d \mu(t) \leq\|f\|_{\infty} \frac{\mu\left(A_{\tau}^{\varepsilon}\right)}{\nu([-\tau, \tau] \backslash I)}+\varepsilon \frac{\mu\left(B_{\tau}^{\varepsilon}\right)}{\nu([-\tau, \tau] \backslash I)} .
$$


By using $\left(\mathbf{H}_{\mathbf{4}}\right)$, it follows that

$$
\limsup _{\tau \rightarrow+\infty} \frac{1}{\nu([-\tau, \tau])} \int_{-\tau}^{+\tau}\left(\sup _{\theta \in[t-r, t]} \mathbb{E}\|f(\theta)\|^{2}\right) d \mu(t) \leq \alpha \varepsilon, \text { for any } \varepsilon>0,
$$

consequently ii) holds.

Thus, we shall show that $i i)$ implies $i i i)$.

Assume that $i i$ ) holds. From the following inequality

$$
\begin{aligned}
\int_{[-\tau, \tau] \backslash I}\left(\sup _{\theta \in[t-r, t]} \mathbb{E}\|f(\theta)\|^{2}\right) d \mu(t) & \geq \int_{A_{\tau}^{\varepsilon}}\left(\sup _{\theta \in[t-r, t]} \mathbb{E}\|f(\theta)\|^{2}\right) d \mu(t) \\
\frac{1}{\nu([-\tau, \tau] \backslash I)} \int_{[-\tau, \tau] \backslash I}\left(\sup _{\theta \in[t-r, t]} \mathbb{E}\|f(\theta)\|^{2}\right) d \mu(t) & \geq \varepsilon \frac{\mu\left(A_{\tau}^{\varepsilon}\right)}{\nu([-\tau, \tau] \backslash I)} \\
\frac{1}{\varepsilon \nu([-\tau, \tau] \backslash I)} \int_{[-\tau, \tau] \backslash I}\left(\sup _{\theta \in[t-r, t]} \mathbb{E}\|f(\theta)\|^{2}\right) d \mu(t) & \geq \frac{\mu\left(A_{\tau}^{\varepsilon}\right)}{\nu([-\tau, \tau] \backslash I)},
\end{aligned}
$$

for $\tau$ sufficiently large, equation (3.2) is obtained, that is $i i i)$.

Definition 3.6. Let $f \in S B C\left(\mathbb{R}, L^{2}(P, H)\right)$ and $\tau \in \mathbb{R}$. We denote by $f_{\tau}$ the function defined by $f_{\tau}(t)=f(t+\tau)$ for $t \in \mathbb{R}$. A subset $\mathfrak{F}$ of $S B C\left(\mathbb{R}, L^{2}(P, H)\right)$ is said to translation invariant if for all $f \in \mathfrak{F}$ we have $f_{\tau} \in \mathfrak{F}$ for all $\tau \in \mathbb{R}$.

Definition 3.7. Let $\mu_{1}$ and $\mu_{2} \in \mathcal{M} . \quad \mu_{1}$ is said to be equivalent to $\mu_{2}\left(\mu_{1} \sim \mu_{2}\right)$ if there exist constants $\alpha$ and $\beta>0$ and a bounded interval $I$ (eventually $I=\emptyset$ ) such that $\alpha \mu_{1}(A) \leq$ $\mu_{2}(A) \leq \beta \mu_{1}(A)$ for $A \in \mathcal{N}$ satisfying $A \cap I=\emptyset$.

Remark 3.8. The relation $\sim$ is an equivalence relation on $\mathcal{M}$.

Theorem 3.9. Let $\mu_{1}, \mu_{2}, \nu_{1}, \nu_{2} \in \mathcal{M}$. If $\mu_{1} \sim \mu_{2}$ and $\nu_{1} \sim \nu_{2}$, then $\mathcal{E}\left(\mathbb{R}, L^{2}(P, H), \mu_{1}, \nu_{1}, r\right)=$ $\mathcal{E}\left(\mathbb{R}, L^{2}(P, H), \mu_{2}, \nu_{2}, r\right)$.

Proof. Since $\mu_{1} \sim \mu_{2}$ and $\nu_{1} \sim \nu_{2}$ there exist some constants $\alpha_{1}, \alpha_{2}, \beta_{1}, \beta_{2}>0$ and a bounded interval I (eventually $I=\emptyset$ ) such that $\alpha_{1} \mu_{1}(A) \leq \mu_{2}(A) \leq \beta_{1} \mu_{1}(A)$ and $\alpha_{2} \nu_{1}(A) \leq \nu_{2}(A) \leq$ $\beta_{2} \nu_{1}(A)$ for each $A \in \mathcal{N}$ satisfies $A \cap I=\emptyset$ i.e

$$
\frac{1}{\beta_{2} \nu_{1}(A)} \leq \frac{1}{\nu_{2}(A)} \leq \frac{1}{\alpha_{2} \nu_{1}(A)}
$$

Since $\mu_{1} \sim \mu_{2}$ and $\mathcal{N}$ is the Lebesgue $\sigma$-field, then for $\tau$ sufficiently large, it follows that

$$
\begin{aligned}
& \frac{\alpha_{1} \mu_{1}\left(\left\{t \in[-\tau, \tau] \backslash I: \sup _{\theta \in[t-r, t]} \mathbb{E}\|f(\theta)\|^{2}>\varepsilon\right\}\right)}{\beta_{2} \nu_{1}([-\tau, \tau] \backslash I)} \leq \\
& \frac{\mu_{2}\left(\left\{t \in[-\tau, \tau] \backslash I: \sup _{\theta \in[t-r, t]} \mathbb{E}\|f(\theta)\|^{2}>\varepsilon\right\}\right)}{\nu_{2}([-\tau, \tau] \backslash I)} \leq \\
& \frac{\beta_{1} \mu_{1}\left(\left\{t \in[-\tau, \tau] \backslash I: \sup _{\theta \in[t-r, t]} \mathbb{E}\|f(\theta)\|^{2}>\varepsilon\right\}\right)}{\alpha_{2} \nu_{1}([-\tau, \tau] \backslash I)}
\end{aligned}
$$

Consequently by Theorem $3.5, \mathcal{E}\left(\mathbb{R}, X, \mu_{1}, \nu_{1}, r\right)=\mathcal{E}\left(\mathbb{R}, X, \mu_{2}, \nu_{2}, r\right)$. 
Let $\mu, \nu \in \mathcal{M}$ denote by

$$
c l(\mu, \nu)=\left\{\omega_{1}, \omega_{2}: \mu \sim \omega_{1} \text { and } \nu \sim \omega_{2}\right\}
$$

Proposition 3.10. [4] Let $\mu \in \mathcal{M}$. Then $\mu$ satisfies $\left(\boldsymbol{H}_{2}\right)$ if and only if the measures $\mu$ and $\mu_{\tau}$ are equivalent for all $\tau \in \mathbb{R}$.

Proposition 3.11. [6] $\left(\boldsymbol{H}_{3}\right)$ implies for all $\sigma$, $\limsup _{\tau \rightarrow \infty} \frac{\mu([-\tau-\sigma, \tau+\sigma])}{\mu([-\tau, \tau])}<+\infty$.

Theorem 3.12. Assume that $\left(\boldsymbol{H}_{\mathcal{2}}\right)$ holds. Then $\mathcal{E}\left(\mathbb{R}, L^{2}(P, H), \mu, \nu, r\right)$ is translation invariant.

Proof. The proof of this theorem is inspired by Theorem (3.5) in [4]. Let $f \in$ $\mathcal{E}\left(\mathbb{R}, L^{2}(P, H), \mu, \nu, r\right)$ and $a \in \mathbb{R}$. Since $\nu(\mathbb{R})=+\infty$, there exists $a_{0}>0$ such that $\nu([-\tau-|a|, \tau+|a|])>0$ for $|a| \geq a_{0}$. Denote by

$$
M_{a}(\tau)=\frac{1}{\nu_{a}([-\tau, \tau])} \int_{[-\tau, \tau]}\left(\sup _{\theta \in[t-r, t]} \mathbb{E}\|f(\theta)\|^{2}\right) d \mu_{a}(t) \forall \tau>0 \text { and } a \in \mathbb{R},
$$

where $\nu_{a}$ is the positive measure defined by equation (3.1). By using Proposition (3.10), it follows that $\nu$ and $\nu_{a}$ are equivalent, $\mu$ and $\mu_{a}$ are equivalent by using Theorem (3.9) we have $\mathcal{E}\left(\mathbb{R}, L^{2}(P, H), \mu_{a}, \nu_{a}, r\right)=\mathcal{E}\left(\mathbb{R}, L^{2}(P, H), \mu, \nu, r\right)$ therefore $f \in \mathcal{E}\left(\mathbb{R}, L^{2}(P, H), \mu_{a}, \nu_{a}, r\right)$ that is $\lim _{\tau \rightarrow+\infty} M_{a}(\tau)=0$ for all $a \in \mathbb{R}$.

For all $A \in \mathcal{N}$, we denote by $\mathcal{X}_{A}$ the characteristic function of $A$. By using definition of the measure $\mu_{a}$, we obtain that

$$
\int_{[-\tau, \tau]} \mathcal{X}_{A}(t) d \mu_{a}(t)=\int_{[-\tau, \tau]} \mathcal{X}_{A}(t) d \mu(t+a)=\int_{[-\tau+a, \tau+a]} d \mu(t) \text { for all } A \in \mathcal{N} .
$$

Since $t \mapsto \sup _{\theta \in[t-r, t]} \mathbb{E}\|f(\theta)\|^{2}$ is the pointwise limit of an increasing sequence of linear combinations of functions, see([ [13]; Theorem 1.17 p.15]), we deduce that

$$
\int_{[-\tau, \tau]} \sup _{\theta \in[t-r, t]} \mathbb{E}\|f(\theta)\|^{2} d \mu_{a}(t)=\int_{[-\tau+a, \tau+a]} \sup _{\theta \in[t-a-r, t-a]} \mathbb{E}\|f(\theta)\|^{2} d \mu(t) .
$$

If we denote by $a^{+}:=\max (a, 0)$ and $a^{-}=\max (-a, 0)$ we have $|a|+a=2 a^{+},|a|-a=2 a^{-}$, and

$[-\tau+a-|a|, \tau+a+|a|]=\left[-\tau-2 a^{-}, \tau+2 a^{+}\right]$. Therefore we obtain

$$
M_{a}(\tau+|a|)=\frac{1}{\nu\left(\left[-\tau-2 a^{-}, \tau+2 a^{+}\right]\right.} \int_{\left[-\tau-2 a^{-}, \tau+2 a^{+}\right]} \sup _{\theta \in[t-a-r, t-a]} \mathbb{E}\|f(\theta)\|^{2} d \mu(t) .
$$

From equation (3.3) and the following inequality

$$
\begin{aligned}
& \frac{1}{\nu([-\tau, \tau])} \int_{[-\tau, \tau]} \sup _{\theta \in[t-a-r, t-a]} \mathbb{E}\|f(\theta)\|^{2} d \mu(t) \leq \\
& \frac{1}{\nu([-\tau, \tau])} \int_{\left[-\tau-2 a^{-}, \tau+2 a^{+}\right]} \sup _{\theta \in[t-a-r, t-a]} \mathbb{E}\|f(\theta)\|^{2} d \mu(t)
\end{aligned}
$$

we obtain

$$
\frac{1}{\nu([-\tau, \tau])} \int_{[-\tau, \tau]} \sup _{\theta \in[t-a-r, t-a]} \mathbb{E}\|f(\theta)\|^{2} d \mu(t) \leq \frac{\nu\left(\left[-\tau-2 a^{-}, \tau+2 a^{+}\right]\right.}{\nu([-\tau, \tau])} \times M_{a}(\tau+|a|) .
$$


This implies,

$$
\frac{1}{\nu([-\tau, \tau])} \int_{[-\tau, \tau]} \sup _{\theta \in[t-a-r, t-a]} \mathbb{E}\|f(\theta)\|^{2} d \mu(t) \leq \frac{\nu([-\tau-2|a|, \tau+2|a|])}{\nu([-\tau, \tau])} \times M_{a}(\tau+|a|) .
$$

From equation (3.3) and equation (3.4) and using Proposition (3.11) we deduce that

$$
\lim _{\tau \rightarrow+\infty} \frac{1}{\nu([-\tau, \tau])} \int_{[-\tau, \tau]} \sup _{\theta \in[t-a-r, t-a]} \mathbb{E}\|f(\theta)\|^{2} d \mu(t)=0
$$

which equivalent to

$$
\lim _{\tau \rightarrow+\infty} \frac{1}{\nu([-\tau, \tau])} \int_{[-\tau, \tau]} \sup _{\theta \in[t-r, t]} \mathbb{E}\|f(\theta-a)\|^{2} d \mu(t)=0
$$

that is $f_{-a} \in \mathcal{E}\left(\mathbb{R}, L^{2}(P, H), \mu, \nu, r\right)$. We have proved that $f \in \mathcal{E}\left(\mathbb{R}, L^{2}(P, H), \mu, \nu, r\right)$ then $f_{-a} \in \mathcal{E}\left(\mathbb{R}, L^{2}(P, H), \mu, \nu, r\right)$ for $a \in \mathbb{R}$. That is $\mathcal{E}\left(\mathbb{R}, L^{2}(P, H), \mu, \nu, r\right)$ is translation invariant.

Proposition 3.13. The space $\operatorname{SPAP}\left(\mathbb{R}, L^{2}(P, H), \mu, \nu, r\right)$ is translation invariant, that is for all $\alpha \in \mathbb{R}$ and $f \in S P A P\left(\mathbb{R}, L^{2}(P, H), \mu, \nu, r\right), f_{\alpha} \in S P A P\left(\mathbb{R}, L^{2}(P, H), \mu, \nu, r\right)$.

\section{Square-Mean $(\mu, \nu)$-Pseudo Almost Periodic Process}

In this section, we define square-mean $(\mu, \nu)$-pseudo almost periodic process and we study their basic properties.

Definition 4.1. Let $f: \mathbb{R} \rightarrow L^{2}(P, H)$ be a continuous stochastic process. $f$ is said be square-mean almost periodic process if for all $\alpha \in \mathbb{R}$, there exists $\tau \in[\alpha, \alpha+l]$ such that

$$
\sup _{t \in \mathbb{R}} \mathbb{E}\|f(t+\tau)-f(t)\|^{2}<\varepsilon
$$

We denote the space of all such stochastic processes by $S A P\left(\mathbb{R}, L^{2}(P, H)\right)$.

Theorem 4.2. [10] The space $\operatorname{SAP}\left(\mathbb{R}, L^{2}(P, H)\right)$ endowed the norm $\|,\|_{\infty}$ is a Banach space.

Definition 4.3. Let $\mu, \nu \in \mathcal{M}$ and $f: \mathbb{R} \rightarrow L^{2}(P, H)$ be a continuous stochastic process. $f$ is said be $(\mu, \nu)$ - square-mean pseudo almost periodic process if it can be decomposed as follows

$$
f=g+\phi
$$

where $g \in S A P\left(\mathbb{R}, L^{2}(P, H)\right)$ and $\phi \in \mathcal{E}\left(\mathbb{R}, L^{2}(P, H), \mu, \nu\right)$.

We denote the space of such stochastic processes by $\operatorname{SPAP}\left(\mathbb{R}, L^{2}(P, H), \mu, \nu\right)$.

Proposition 4.4. [7] Assume that $\left(\boldsymbol{H}_{3}\right)$ holds. Then the decomposition of $(\mu, \nu)$-pseudo almost periodic function in the form $f=g+\phi$ where $g \in A P(\mathbb{R}, X)$ and $\phi \in \mathcal{E}(\mathbb{R}, X, \mu, \nu)$ is unique.

Proposition 4.5. [14] Let $\mu, \nu \in \mathcal{M}$. Assume $\left(\boldsymbol{H}_{3}\right)$ holds. Then the decomposition of a $(\mu, \nu)$ pseudo almost periodic function $\phi=\phi_{1}+\phi_{2}$, where $\phi_{1} \in A P(\mathbb{R}, X)$ and $\phi_{2} \in \mathcal{E}(\mathbb{R}, X, \mu, \nu)$ is unique.

Remark 4.6. Let $X=L^{2}(P, H)$. Then the Proposition (4.4) always holds. 
Definition 4.7. Let $\mu, \nu \in \mathcal{M}$ and $f: \mathbb{R} \rightarrow L^{2}(P, H)$ be a continuous stochastic process.

$f$ is said be $(\mu, \nu)$ - square-mean pseudo almost periodic process of class $r$ if it can be decomposed as follows

$$
f=g+\phi
$$

where $g \in S A P\left(\mathbb{R}, L^{2}(P, H)\right)$ and $\phi \in \mathcal{E}\left(\mathbb{R}, L^{2}(P, H), \mu, \nu, r\right)$.

We denote the space of such stochastic processes by $\operatorname{SPAP}\left(\mathbb{R}, L^{2}(P, H), \mu, \nu, r\right)$.

Proposition 4.8. $S P A P\left(\mathbb{R}, L^{2}(P, H), \mu, \nu, r\right)$ is a Banach space.

Proof. This proposition is a consequence of Theorem(4.2) and Proposition(3.4).

Proposition 4.9. [14] Let $\mu, \nu \in \mathcal{M}$ and assume $\left(\boldsymbol{H}_{3}\right)$ holds. Then the decomposition of $(\mu, \nu)$-pseudo almost periodic function $\phi=\phi_{1}+\phi_{2}$, where $\phi \in A P\left(\mathbb{R}, L^{2}(P, H)\right)$ and $\phi_{2} \in$ $\mathcal{E}\left(\mathbb{R}, L^{2}(P, H), \mu, \nu, r\right)$ is unique.

Proposition 4.10. Let $\mu_{1}, \mu_{2}, \nu_{1}$ and $\nu_{2} \in \mathcal{M}$ if $\mu_{1} \sim \mu_{2}$ and $\nu_{1} \sim \nu_{2}$, then $S P A P\left(\mathbb{R}, L^{2}(P, H), \mu_{1}, \nu_{1}, r\right)=S P A P\left(\mathbb{R} ; L^{2}(P, H), \mu_{2}, \nu_{2}, r\right)$.

This Proposition is a consequence of Theorem(3.9).

Theorem 4.11. Assume that $\left(\boldsymbol{H}_{3}\right)$ holds. Let $\mu, \nu \in \mathcal{M}$ and $\phi \in S P A P\left(\mathbb{R}, L^{2}(P, H), \mu, \nu, r\right)$ then the function $t \rightarrow \phi_{t}$, belongs to $\operatorname{SPAP}\left(C\left([-r, 0], L^{2}(P, H)\right), \mu, \nu, r\right)$.

Proof. Assume that $\phi=g+h$, where $g \in S A P\left(\mathbb{R}, L^{2}(P, H)\right)$ and $h \in \mathcal{E}\left(\mathbb{R}, L^{2}(P, H), \mu, \nu, r\right)$. Then we can see that, $\phi_{t}=g_{t}+h_{t}$ and $g_{t}$ is square mean almost periodic process. Let us denote by

$$
M_{\alpha}(\tau)=\frac{1}{\nu_{\alpha}([-\tau, \tau])} \int_{-\tau}^{\tau}\left(\sup _{\theta \in[t-r, t]} \mathbb{E}\|h(\theta)\|^{2}\right) d \mu_{\alpha}(t) .
$$

Where $\mu_{\alpha}$ and $\nu_{\alpha}$ are the positive measures defined by equation (3.1). By using Proposition (3.10), it follows that $\mu_{\alpha}$ and $\mu$ are equivalent and $\nu_{\alpha}$ and $\nu$ are also equivalent. Then by using Theorem (4.10) we have $\mathcal{E}\left(\mathbb{R}, L^{2}(P, H), \mu_{\alpha}, \nu_{\alpha}, r\right)=\mathcal{E}\left(\mathbb{R}, L^{2}(P, H), \mu, \nu, r\right)$ therefore $h \in$ $\mathcal{E}\left(\mathbb{R}, L^{2}(P, H), \mu_{\alpha}, \nu_{\alpha}, r\right)$ that is $\lim _{\tau \rightarrow+\infty} M_{\alpha}(\tau)=0$ for all $\alpha \in \mathbb{R}$. On the other hand, for $r>0$ we have

$$
\begin{aligned}
\frac{1}{\nu([-\tau, \tau])} & \int_{-\tau}^{\tau}\left(\sup _{\theta \in[t-r, t]}\left(\sup _{\eta \in[-r, 0]}\left(\mathbb{E}\|h(\theta+\eta)\|^{2}\right)\right) d \mu(t) \leq \frac{1}{\nu([-\tau, \tau])} \int_{-\tau}^{\tau} \sup _{\theta \in[t-2 r, t]}\left(\mathbb{E}\|h(\theta)\|^{2}\right) d \mu(t)\right. \\
\leq & \frac{1}{\nu([-\tau, \tau])} \int_{-\tau}^{\tau}\left[\sup _{\theta \in[t-2 r, t-r]}\left(\mathbb{E}\|h(\theta)\|^{2}\right)+\sup _{\theta \in[t-r, t]}\left(\mathbb{E}\|h(\theta)\|^{2}\right)\right] d \mu(t) \\
\leq & \frac{1}{\nu([-\tau, \tau])} \int_{-\tau}^{\tau} \sup _{\theta \in[-\tau-r, \tau+r]}\left(\mathbb{E}\|h(\theta)\|^{2}\right) d \mu(t+r)+\frac{1}{\nu([-\tau, \tau])} \int_{-\tau}^{\tau} \sup _{\theta \in[t-r, t]}\left(\mathbb{E}\|h(\theta)\|^{2}\right) d \mu(t) \\
\leq & \frac{\nu([-\tau-r, \tau+r])}{\nu([-\tau, \tau])} \times \frac{1}{\nu([-\tau-r, \tau+r])} \int_{-\tau-r}^{\tau+r} \sup _{\theta \in[t-r, t]} \mathbb{E}\|h(\theta)\|^{2} d \mu(t+r) \\
+ & \frac{1}{\nu([-\tau, \tau])} \int_{-\tau}^{\tau} \sup _{\theta \in[t-r, t]} \mathbb{E}\|h(\theta)\|^{2} d \mu(t)
\end{aligned}
$$

Consequently,

$$
\begin{aligned}
\frac{1}{\nu([-\tau, \tau])} \int_{-\tau}^{\tau} \sup _{\theta \in[t-r, t]}\left(\sup _{\eta \in[-r, 0]}\left(\mathbb{E}\|h(\theta+\eta)\|^{2}\right)\right) d \mu(t) & \leq \frac{\nu([-\tau-r, \tau+r])}{\nu([-\tau, \tau])} \times M_{r}(\tau+r) \\
& +\frac{1}{\nu([-\tau, \tau])} \int_{-\tau}^{\tau} \sup _{\theta \in[t-r, t]} \mathbb{E}\|h(\theta)\|^{2} d \mu(t)
\end{aligned}
$$


Using Proposition(3.11), and Proposition(3.10), it follows that, $\left.\phi_{t} \in S P A P\left(C[-r, 0], L^{2}(P, H)\right), \mu, \nu, r\right)$. Thus, we obtain the desired result

Next, we study the composition of the space square-mean $(\mu, \nu)$-pseudo almost periodic process.

Definition 4.12. [10] Let $f: \mathbb{R} \times L^{2}(P, H) \rightarrow L^{2}(P, H),(t, x) \mapsto f(t, x)$ be continuous. $f$ is said be square-mean almost periodic in $t \in \mathbb{R}$ uniformly in $x \in L^{2}(P, H)$ if for all compact $K$ of $L^{2}(P, H)$ and for any $\varepsilon>0$ there exists $l(\varepsilon, K)$ such that for all $\alpha \in \mathbb{R}$, there exists $\tau \in[\alpha, \alpha+l(\varepsilon, K)]$ with

$$
x \in K, \quad \sup _{t \in \mathbb{R}} \mathbb{E}\|f(t+\tau, x)-f(t, x)\|^{2}<\varepsilon .
$$

We denote the following space of stochastic processes by $S A P\left(\mathbb{R} \times L^{2}(P, H), L^{2}(P, H)\right)$.

Theorem 4.13. [10] Let $f: \mathbb{R} \times L^{2}(P, H) \rightarrow L^{2}(P, H),(t, x) \mapsto f(t, x)$ be a square almost periodic process in $t$ uniformly in $x \in L^{2}(P, H)$. Suppose that $f$ is Lipschitz in the following sense: there exists a positive number $L$ such that for any $x, y \in L^{2}(P, H)$,

$$
\mathbb{E}\|f(t, x)-f(t, y)\|^{2} \leq L \cdot \mathbb{E}\|x-y\|^{2} .
$$

Then $t \mapsto f(t, x(t)) \in S A P\left(\mathbb{R}, L^{2}(P, H)\right)$ for any $x \in S A P\left(\mathbb{R}, L^{2}(P, H)\right)$.

Definition 4.14. Let $\mu, \nu \in \mathcal{M}$. A continuous functions $f(t, x): \mathbb{R} \times L^{2}(P, H) \rightarrow L^{2}(P, H)$ is said to be square-mean $(\mu, \nu)$-pseudo almost periodic of class $r$ in $t$ for any $x \in L^{2}(P, H)$ if it can be decomposed as $f=g+\phi$, where $g \in S A P\left(\mathbb{R} \times L^{2}(P, H), L^{2}(P, H)\right), \phi \in \mathcal{E}(\mathbb{R} \times$ $\left.L^{2}(P, H), L^{2}(P, H), \mu, \nu, r\right)$. Denote the set of all such stochastically continuous processes by $S P A P\left(\mathbb{R} \times L^{2}(P, H), L^{2}(P, H), \mu, \nu, r\right)$.

Proposition 4.15. Let $a_{i} \in \mathbb{R}, i \in \mathbb{N}$. Then $\left|\sum_{i=1}^{n} a_{i}\right|^{2} \leq n \sum_{i=1}^{n}\left|a_{i}\right|^{2}$.

Theorem 4.16. Let $\mu, \nu \in \mathcal{M}$ satisfy $\left(\boldsymbol{H}_{2}\right)$. Suppose that $f \in S P A P(\mathbb{R} \times$ $\left.L^{2}(P, H), L^{2}(P, H), \mu, \nu, r\right)$ and that there exists a positive number $L$ such that, for any $x, y \in$ $L^{2}(P, H)$,

$$
\mathbb{E}\|f(t, x)-f(t, y)\|^{2} \leq L \cdot \mathbb{E}\|x-y\|^{2}
$$

for $t \in \mathbb{R}$. Then $t \mapsto f(t, x(t)) \in \operatorname{SPAP}\left(\mathbb{R}, L^{2}(P, H), \mu, \nu, r\right)$ for any $x \in$ $S P A P\left(\mathbb{R} ; L^{2}(P, H), \mu, \nu, r\right)$.

Proof. Since $x \in S P A P\left(\mathbb{R} ; L^{2}(P, H), \mu, \nu, r\right)$, then we can decompose $x=x_{1}+x_{2}$, where $x_{1} \in S A P\left(\mathbb{R}, L^{2}(P, H)\right)$ and $x_{2} \in \mathcal{E}\left(\mathbb{R}, L^{2}(P, H), \mu, \nu, r\right)$.

Otherwise, since $f \in S P A P\left(\mathbb{R} \times L^{2}(P, H), L^{2}(P, H), \mu, \nu, r\right)$, then $f=f_{1}+f_{2}$, where $f_{1} \in$ $S A P\left(\mathbb{R} \times L^{2}(P, H), L^{2}(P, H)\right)$ and $f_{2} \in \mathcal{E}\left(\mathbb{R} \times L^{2}(P, H), L^{2}(P, H), \mu, \nu, r\right)$.

The function $f$ can be decomposed as

$$
\begin{aligned}
f(t, x(t)) & =f_{1}\left(t, x_{1}(t)\right)+\left[f(t, x(t))-f\left(t, x_{1}(t)\right)\right]+\left[f\left(t, x_{1}(t)\right)-f_{1}\left(t, x_{1}(t)\right)\right] \\
& =f_{1}\left(t, x_{1}(t)\right)+\left[f(t, x(t))-f\left(t, x_{1}(t)\right)\right]+f_{2}\left(t, x_{1}(t)\right)
\end{aligned}
$$

Using Theorem (4.13), we have $\left(t \rightarrow f_{1}\left(t, x_{1}(t)\right) \in S A P\left(\mathbb{R} \times L^{2}(P, H), L^{2}(P, H)\right)\right.$.

It remains to show that the both functions $t \rightarrow\left[f(t, x(t))-f\left(t, x_{1}(t)\right)\right]$ and $t \rightarrow f_{2}\left(t, x_{1}(t)\right)$ 
belong to $\mathcal{E}\left(\mathbb{R} \times L^{2}(P, H), L^{2}(P, H), \mu, \nu, r\right)$.

We have,

$$
\begin{aligned}
\mathbb{E}\left\|f(t, x(t))-f\left(t, x_{1}(t)\right)\right\|^{2} & \leq L \cdot \mathbb{E}\left\|x(t)-x_{1}(t)\right\|^{2} \\
\sup _{\theta \in[t-r, t]} \mathbb{E} \| f(\theta, x(\theta))-f\left(\theta, x_{1}(\theta) \|^{2}\right. & \leq L \sup _{\theta \in[t-r, t]} \mathbb{E}\left\|x(\theta)-x_{1}(\theta)\right\|^{2} .
\end{aligned}
$$

It follows that

$$
\begin{aligned}
& \frac{1}{\nu([-\tau, \tau])} \int_{[-\tau, \tau]} \sup _{\theta \in[t-r, t]} \mathbb{E} \| f(\theta, x(\theta))-f\left(\theta, x_{1}(\theta) \|^{2} d \mu(t) \leq\right. \\
& \frac{L}{\nu([-\tau, \tau])} \int_{[-\tau, \tau]} \sup _{\theta \in[t-r, t]} \mathbb{E}\left\|x(\theta)-x_{1}(\theta)\right\|^{2} d \mu(t) \leq \\
& \frac{L}{\nu([-\tau, \tau])} \int_{[-\tau, \tau]} \sup _{\theta \in[t-r, t]} \mathbb{E}\left\|x_{2}(\theta)\right\|^{2} d \mu(t) .
\end{aligned}
$$

Since $x_{2} \in \mathcal{E}\left(\mathbb{R}, L^{2}(P, H), \mu, \nu, r\right)$ then $t \rightarrow f(t, x(t))-f\left(t, x_{1}(t)\right)$ is $(\mu, \nu)$-ergodic

Now to complete the proof, it is enough to prove $t \rightarrow f_{2}\left(t, x_{1}(t)\right)$ is $(\mu, \nu)$-ergodic. Since $f_{2}$ is uniformly continuous on the compact set $K=\left\{x_{1}(t): t \in \mathbb{R}\right\}$ with respect to the second variable $x$, we deduce that for given $\varepsilon$, there exists $\delta>0$ such that for all $t \in \mathbb{R}, \zeta_{1}$ and $\zeta_{2} \in K$, one has

$$
\left\|\zeta_{1}-\zeta_{2}\right\| \leq \delta \Longrightarrow\left\|f_{2}\left(t, \zeta_{1}\right)-f_{2}\left(t, \zeta_{2}\right)\right\| \leq \varepsilon
$$

Therefore, there exist $n(\varepsilon) \in \mathbb{N}$ and $\left\{x_{i}\right\}_{i=1}^{n(\varepsilon)} \subset K$, such that

$$
K \subset \bigcup_{i=1}^{n(\varepsilon)} B\left(x_{i}, \delta\right)
$$

then

$$
\begin{aligned}
\left\|f_{2}\left(t, x_{1}(t)\right)\right\| & \leq \varepsilon+\sum_{i=1}^{n(\varepsilon)}\left\|f_{2}\left(t, x_{i}\right)\right\| \\
\left\|f_{2}\left(t, x_{1}(t)\right)\right\|^{2} & \leq\left(\varepsilon+\sum_{i=1}^{n(\varepsilon)}\left\|f_{2}\left(t, x_{i}\right)\right\|\right)^{2} \\
& \leq 2\left(\varepsilon^{2}+\left(\sum_{i=1}^{n(\varepsilon)}\left\|f_{2}\left(t, x_{i}\right)\right\|\right)^{2}\right)
\end{aligned}
$$

By using the Proposition (4.15), we have

$$
\left\|f_{2}\left(t, x_{1}(t)\right)\right\|^{2} \leq 2\left(\varepsilon+n(\varepsilon) \sum_{i=1}^{n(\varepsilon)}\left\|f_{2}\left(t, x_{i}\right)\right\|^{2}\right)
$$


It follows that

$$
\begin{aligned}
& \frac{1}{\nu([-\tau, \tau])} \int_{[-\tau, \tau]} \sup _{\theta \in[t-r, t]} \mathbb{E}\left\|f_{2}\left(\theta, x_{1}(\theta)\right)\right\|^{2} d \mu(t) \leq \\
& 2\left(\frac{\varepsilon \mu([-\tau, \tau]}{\nu([-\tau, \tau])}+n(\varepsilon) \sum_{i=1}^{n(\varepsilon)} \frac{1}{\nu([-\tau, \tau])} \int_{[-\tau, \tau]} \sup _{\theta \in[t-r, t]} \mathbb{E}\left\|f_{2}\left(\theta, x_{i}\right)\right\|^{2} d \mu(t)\right) .
\end{aligned}
$$

By the fact that

$$
\forall i \in\{1, \ldots, n(\varepsilon)\}, \quad \lim _{\tau \rightarrow+\infty} \frac{1}{\nu([-\tau, \tau])} \int_{-\tau}^{+\tau}\left(\sup _{\theta \in[t-r, t]} \mathbb{E}\left\|f_{2}\left(\theta, x_{i}\right)\right\|^{2}\right) d \mu(t)=0
$$

we deduce that

$$
\forall \varepsilon>0, \limsup _{\tau \rightarrow+\infty} \frac{1}{\nu([-\tau, \tau])} \int_{-\tau}^{+\tau}\left(\sup _{\theta \in[t-r, t]} \mathbb{E}\left\|f_{2}\left(\theta, x_{1}(\theta)\right)\right\|^{2}\right) d \mu(t) \leq 2 \alpha \varepsilon .
$$

Therefore $t \rightarrow f_{2}\left(t, x_{1}(t)\right)$ is ergodic and the theorem is proved.

$\left(\mathbf{H}_{\mathbf{5}}\right)$ : $\mathrm{g}$ is a stochastically bounded process.

Theorem 4.17. Assume that $\left(\boldsymbol{H}_{\boldsymbol{O}}\right),\left(\boldsymbol{H}_{\mathbf{1}}\right),\left(\boldsymbol{H}_{4}\right)$ and $\left(\boldsymbol{H}_{\boldsymbol{5}}\right)$ hold and the semigroup $(\mathcal{U}(t))_{t \geq 0}$ is hyperbolic. If $f$ is bounded and continuous on $\mathbb{R}$, then there exists a unique bounded solution $u$ of equation (1.1) on $\mathbb{R}$ given by

$$
\begin{aligned}
u_{t} & =\lim _{\lambda \rightarrow+\infty} \int_{-\infty}^{t} \mathcal{U}^{s}(t-s) \Pi^{s}\left(\widetilde{B}_{\lambda} X_{0} f(s)\right) d s+\lim _{\lambda \rightarrow+\infty} \int_{+\infty}^{t} \mathcal{U}^{u}(t-s) \Pi^{u}\left(\widetilde{B}_{\lambda} X_{0} f(s)\right) d s \\
& +\lim _{\lambda \rightarrow+\infty} \int_{-\infty}^{t} \mathcal{U}^{s}(t-s) \Pi^{s}\left(\widetilde{B}_{\lambda} X_{0} g(s)\right) d W(s)+\lim _{\lambda \rightarrow+\infty} \int_{+\infty}^{t} \mathcal{U}^{u}(t-s) \Pi^{u}\left(\widetilde{B}_{\lambda} X_{0} g(s)\right) d W(s)
\end{aligned}
$$

$\forall t \geq 0$, where $\widetilde{B}_{\lambda}=\lambda\left(\lambda I-\widetilde{\mathcal{A}}_{\mathcal{U}}\right)^{-1}, \Pi^{s}$ and $\Pi^{u}$ are the projections of $C_{0}$ onto the stable and unstable subspaces.

Proof. Let

$$
\begin{aligned}
u_{t}= & v(t)+\lim _{\lambda \rightarrow+\infty} \int_{-\infty}^{t} \mathcal{U}^{s}(t-s) \Pi^{s}\left(\widetilde{B}_{\lambda} X_{0} g(s)\right) d W(s) \\
& +\lim _{\lambda \rightarrow+\infty} \int_{+\infty}^{t} \mathcal{U}^{u}(t-s) \Pi^{u}\left(\widetilde{B}_{\lambda} X_{0} g(s)\right) d W(s) \forall t \geq 0,
\end{aligned}
$$

where

$$
v(t)=\lim _{\lambda \rightarrow+\infty} \int_{-\infty}^{t} \mathcal{U}^{s}(t-s) \Pi^{s}\left(\widetilde{B}_{\lambda} X_{0} f(s)\right) d s+\lim _{\lambda \rightarrow+\infty} \int_{+\infty}^{t} \mathcal{U}^{u}(t-s) \Pi^{u}\left(\widetilde{B}_{\lambda} X_{0} f(s)\right) d s
$$

Let us first prove that $u_{t}$ exists. The existence of $v(t)$ have proved by [1]. Now, we show that the limit $\lim _{\lambda \rightarrow+\infty} \int_{-\infty}^{t} \mathcal{U}^{s}(t-s) \Pi^{s} \widetilde{B}_{\lambda}\left(X_{0} g(s)\right) d W(s)$ exist.

For $t \in \mathbb{R}$ we have, 


$$
\begin{aligned}
\mathbb{E}\left\|\int_{-\infty}^{t} \mathcal{U}^{s}(t-s) \Pi^{s}\left(\widetilde{B}_{\lambda} X_{0} g(s)\right) d W(s)\right\|^{2} & \leq \mathbb{E}\left(\int_{-\infty}^{t} \bar{M}^{2} e^{-2 w(t-s)}\left|\Pi^{s}\right|^{2}\left\|\widetilde{B}_{\lambda}\left(X_{0} g(s)\right)\right\|^{2} d s\right) \\
& \leq \bar{M}^{2} \mathbb{E}\left(\int_{-\infty}^{t} e^{-2 w(t-s)}\left|\Pi^{s}\right|^{2}\left\|\widetilde{B}_{\lambda}\left(X_{0} g(s)\right)\right\|^{2} d s\right) \\
& \leq \bar{M}^{2} \widetilde{M}^{2}\left|\Pi^{s}\right|^{2} \mathbb{E}\left(\int_{-\infty}^{t} e^{-2 w(t-s)}\|g(s)\|^{2} d s\right) \\
& \leq \bar{M}^{2} \widetilde{M}^{2}\left|\Pi^{s}\right|^{2} \sum_{n=1}^{\infty} \mathbb{E}\left(\int_{t-n}^{t-n+1} e^{-2 w(t-s)}\|g(s)\|^{2} d s\right) .
\end{aligned}
$$

Using the Hölder inequality, we obtain

$$
\begin{aligned}
& \mathbb{E}\left\|\int_{-\infty}^{t} \mathcal{U}^{s}(t-s) \Pi^{s} \widetilde{B}_{\lambda}\left(X_{0} g(s)\right) d W(s)\right\|^{2} \leq \\
& \bar{M}^{2} \widetilde{M}^{2}\left|\Pi^{s}\right|^{2} \sum_{n=1}^{+\infty}\left(\int_{t-n}^{t-n+1} e^{-4 w(t-s)} d s\right)^{\frac{1}{2}} \mathbb{E}\left(\int_{t-n}^{t-n+1}\|g(s)\|^{2} d s\right)^{\frac{1}{2}} \leq \\
& \bar{M}^{2} \widetilde{M}^{2}\left|\Pi^{s}\right|^{2} \frac{1}{2 \sqrt{w}} \sum_{n=1}^{\infty}\left(e^{-4 w(n-1)}-e^{-4 w n}\right)^{\frac{1}{2}} \mathbb{E}\left(\int_{t-n}^{t-n+1}\|g(s)\|^{2} d s\right)^{\frac{1}{2}} \leq \\
& \bar{M}^{2} \widetilde{M}^{2}\left|\Pi^{s}\right|^{2} \frac{1}{2 \sqrt{w}}\left(e^{4 w n}-1\right)^{\frac{1}{2}} \sum_{n=1}^{\infty} e^{-2 w n} \times \mathbb{E}\left(\int_{t-n}^{t-n+1}\|g(s)\|^{2} d s\right)^{\frac{1}{2}} .
\end{aligned}
$$

Since the serie $\sum_{n=1}^{\infty} e^{-2 w n}$ is convergent, then it exists a constant $c>0$ such that $\sum_{n=1}^{\infty} e^{-2 w n} \leq c$, moreover it follows that

$$
\begin{aligned}
& \mathbb{E}\left\|\int_{-\infty}^{t} \mathcal{U}^{s}(t-s) \Pi^{s}\left(\widetilde{B}_{\lambda} X_{0} g(s)\right) d W(s)\right\|^{2} \leq \\
& \bar{M} \widetilde{M}^{2}\left|\Pi^{s}\right|^{2} \frac{1}{2 \sqrt{w}}\left(e^{4 w}-1\right)^{\frac{1}{2}} \mathbb{E}\|g(s)\| \sum_{n=1}^{\infty} e^{-2 w n} \\
& \leq \quad \gamma \sum_{n=1}^{\infty} e^{-2 w n} \\
& \leq \quad \gamma c,
\end{aligned}
$$

where, $\gamma=\bar{M}^{2} \widetilde{M}^{2}\left|\Pi^{s}\right|^{2} \frac{1}{2 \sqrt{w}}\left(e^{4 w}-1\right)^{\frac{1}{2}} \mathbb{E}\|g(s)\|$.

Let $F(n, s, t)=\mathcal{U}^{s}(t-s) \Pi^{s}\left(\widetilde{B}_{\lambda} X_{0} g(s)\right)$ for $n \in \mathbb{N}$ for $s \leq t$.

For $n$ is sufficiently large and $\sigma \leq t$, we have

$$
\begin{aligned}
& \mathbb{E}\left\|\int_{-\infty}^{\sigma} F(n, s, t) d W(s)\right\|^{2} \leq \\
& \bar{M}^{2} \widetilde{M}^{2}\left|\Pi^{s}\right|^{2} \sum_{n=1}^{+\infty}\left(\int_{\sigma-n}^{\sigma-n+1} e^{-4 w(t-s)} d s\right)^{\frac{1}{2}} \times \mathbb{E}\left(\int_{\sigma-n}^{\sigma-n+1}\|g(s)\|^{2} d s\right)^{\frac{1}{2}} \leq
\end{aligned}
$$




$$
\begin{aligned}
& \left.\bar{M}^{2} \widetilde{M}^{2}\left|\Pi^{s}\right|^{2} \frac{1}{2 \sqrt{\omega}}\left(\sum_{n=1}^{\infty}\left(e^{-4 \omega(t-\sigma+n-1)}-e^{-4 \omega(t-\sigma+n)}\right)^{\frac{1}{2}} \times \mathbb{E}\left(\int_{\sigma-n}^{\sigma-n+1}\|g(s)\|^{2} d s\right)^{\frac{1}{2}}\right)\right) \leq \\
& \left.\bar{M}^{2} \widetilde{M}^{2}\left|\Pi^{s}\right|^{2} \frac{1}{2 \sqrt{\omega}} e^{-2 \omega(t-\sigma)}\left(e^{4 \omega}-1\right)^{\frac{1}{2}} \sum_{n=1}^{\infty} e^{-2 \omega n} \mathbb{E}\left(\int_{\sigma-n}^{\sigma-n+1}\|g(s)\|^{2} d s\right)^{\frac{1}{2}}\right) \leq \gamma c e^{-2 w(t-\sigma)}
\end{aligned}
$$

It follows that for $n$ and $m$ sufficiently large and $\sigma \leq t$, we have

$$
\begin{aligned}
& \mathbb{E}\left\|\int_{-\infty}^{t} F(n, s, t) d W(s)-\int_{\infty}^{t} F(m, s, t) d W(s)\right\|^{2} \leq \\
& \mathbb{E} \| \int_{-\infty}^{\sigma} F(n, s, t) d W(s)+\int_{\sigma}^{t} F(n, s, t) d W(s)-\int_{-\infty}^{\sigma} F(m, s, t) d W(s) \\
& -\int_{\sigma}^{t} F(m, s, t) d W(s) \|^{2} \leq \\
& 3 \mathbb{E}\left\|\int_{-\infty}^{\sigma} F(n, s, t) d W(s)\right\|^{2}+3 \mathbb{E}\left\|\int_{-\infty}^{\sigma} F(m, s, t) d W(s)\right\| \|^{2} \\
& +3 \mathbb{E}\left\|\int_{\sigma}^{t} F(n, s, t) d W(s)-\int_{\sigma}^{t} F(m, s, t) d W(s)\right\|^{2} \leq \\
& 6 \gamma c e^{-2 \omega(t-\sigma)}+3 \mathbb{E}\left\|\int_{\sigma}^{t} F(n, s, t) d W(s)-\int_{\sigma}^{t} F(m, s, t) d W(s)\right\|^{2}
\end{aligned}
$$

Since $\lim _{n \rightarrow+\infty} \mathbb{E}\left\|\int_{\sigma}^{t} F(n, s, t) d W(s)\right\|^{2}$ exists, then

$$
\limsup _{n, m \rightarrow+\infty} \mathbb{E}\left\|\int_{-\infty}^{t} F(n, s, t) d W(s)-\int_{-\infty}^{t} F(m, s, t) d W(s)\right\|^{2} \leq 6 \gamma c e^{-2 \omega(t-\sigma)}
$$

If $\sigma \rightarrow-\infty$, then

$$
\limsup _{n, m \rightarrow+\infty} \mathbb{E}\left\|\int_{-\infty}^{t} F(n, s, t) d W(s)-\int_{-\infty}^{t} F(m, s, t) d W(s)\right\|^{2}=0 .
$$

We deduce that the limit

$$
\lim _{n \rightarrow+\infty} \mathbb{E}\left\|\int_{-\infty}^{t} F(n, s, t) d W(s)\right\|^{2}=\lim _{n \rightarrow+\infty} \mathbb{E}\left\|\int_{-\infty}^{t} \mathcal{U}^{s}(t-s) \Pi^{s}\left(\widetilde{B}_{n} X_{0} g(s)\right) d W(s)\right\|^{2}
$$

exists. Therefore, $\lim _{n \rightarrow+\infty} \int_{-\infty}^{t} \mathcal{U}^{s}(t-s) \Pi^{s}\left(\widetilde{B}_{n} X_{0} g(s)\right) d W(s)$ exists. In addition, one can show that the function

$$
t \rightarrow \lim _{n \rightarrow+\infty} \mathbb{E} \| \int_{-\infty}^{t} \mathcal{U}^{s}(t-s) \Pi^{s}\left(\widetilde{B}_{n} X_{0} g(s) d s \|^{2}\right.
$$

is bounded on $\mathbb{R}$. Similary, we can show that the function

$$
t \rightarrow \lim _{n \rightarrow+\infty} \int_{t}^{+\infty} \mathcal{U}^{u}(t-s) \Pi^{u}\left(\widetilde{B}_{n} X_{0} g(s)\right) d W(s)
$$

is well defined and bounded on $\mathbb{R}$.

Proposition 4.18. [14] A function $\phi \in C(\mathbb{R}, X)$ is almost periodic if and only if the space of functions $\left\{\phi_{\tau}: \tau \in \mathbb{R}\right\}$, where $\phi_{\tau}(t)=\phi(t+\tau)$, is relatively compact in $B C(\mathbb{R} ; X)$

Remark 4.19. As $L^{2}(P, H)$ is a space Banach then the Proposition(4.18) holds. 
Theorem 4.20. Assume that $\left(\boldsymbol{H}_{\boldsymbol{5}}\right)$. Let $f, g \in S A P\left(\mathbb{R} ; L^{2}(P, H)\right)$ and $\Gamma$ be the mapping defined for $t \in \mathbb{R}$ by

$$
\begin{aligned}
\Gamma(f, g)(t) & =\lim _{\lambda \rightarrow+\infty} \int_{-\infty}^{t} \mathcal{U}^{s}(t-s) \Pi^{s}\left(\widetilde{B}_{\lambda} X_{0} f(s)\right) d s+\lim _{\lambda \rightarrow+\infty} \int_{+\infty}^{t} \mathcal{U}^{u}(t-s) \Pi^{u}\left(\widetilde{B}_{\lambda} X_{0} f(s)\right) d s \\
& +\lim _{\lambda \rightarrow+\infty} \int_{-\infty}^{t} \mathcal{U}^{s}(t-s) \Pi^{s}\left(\widetilde{B}_{\lambda} X_{0} g(s)\right) d W(s) \\
& +\lim _{\lambda \rightarrow+\infty} \int_{+\infty}^{t} \mathcal{U}^{u}(t-s) \Pi^{u}\left(\widetilde{B}_{\lambda} X_{0} g(s)\right) d W(s)
\end{aligned}
$$

Then $\Gamma(f, g) \in S A P\left(\mathbb{R} ; L^{2}(P, H)\right)$.

Proof. $\Gamma(f, g)_{\tau}(t)=\Gamma(f, g)(t+\tau)$

$$
\begin{aligned}
& =\lim _{\lambda \rightarrow+\infty} \int_{-\infty}^{t+\tau} \mathcal{U}^{s}(t+\tau-s) \Pi^{s}\left(\widetilde{B}_{\lambda} X_{0} f(s)\right) d s+\lim _{\lambda \rightarrow+\infty} \int_{+\infty}^{t+\tau} \mathcal{U}^{u}(t+\tau-s) \Pi^{u}\left(\widetilde{B}_{\lambda} X_{0} f(s)\right) d s \\
& +\lim _{\lambda \rightarrow+\infty} \int_{-\infty}^{t+\tau} \mathcal{U}^{s}(t+\tau-s) \Pi^{s}\left(\widetilde{B}_{\lambda} X_{0} g(s)\right) d W(s)+\lim _{\lambda \rightarrow+\infty} \int_{+\infty}^{t+\tau} \mathcal{U}^{u}(t+\tau-s) \Pi^{u}\left(\widetilde{B}_{\lambda} X_{0} g(s)\right) d W(s) \\
& =\lim _{\lambda \rightarrow+\infty} \int_{-\infty}^{t} \mathcal{U}^{s}(t-s) \Pi^{s}\left(\widetilde{B}_{\lambda} X_{0} f(s+\tau)\right) d s+\lim _{\lambda \rightarrow+\infty} \int_{+\infty}^{t} \mathcal{U}^{u}(t-s) \Pi^{u}\left(\widetilde{B}_{\lambda} X_{0} f(s+\tau)\right) d s \\
& +\lim _{\lambda \rightarrow+\infty} \int_{-\infty}^{t} \mathcal{U}^{s}(t-s) \Pi^{s}\left(\widetilde{B}_{\lambda} X_{0} g(s+\tau)\right) d W(s)+\lim _{\lambda \rightarrow+\infty} \int_{+\infty}^{t} \mathcal{U}^{u}(t-s) \Pi^{u}\left(\widetilde{B}_{\lambda} X_{0} g(s+\tau)\right) d W(s) \\
& =\lim _{\lambda \rightarrow+\infty} \int_{-\infty}^{t} \mathcal{U}^{s}(t-s) \Pi^{s}\left(\widetilde{B}_{\lambda} X_{0} f_{\tau}(s)\right) d s+\lim _{\lambda \rightarrow+\infty} \int_{+\infty}^{t} \mathcal{U}^{u}(t-s) \Pi^{u}\left(\widetilde{B}_{\lambda} X_{0} f_{\tau}(s)\right) d s \\
& +\lim _{\lambda \rightarrow+\infty} \int_{-\infty}^{t} \mathcal{U}^{s}(t-s) \Pi^{s}\left(\widetilde{B}_{\lambda} X_{0} g_{\tau}(s)\right) d W(s)+\lim _{\lambda \rightarrow+\infty} \int_{+\infty}^{t} \mathcal{U}^{u}(t-s) \Pi^{u}\left(\widetilde{B}_{\lambda} X_{0} g_{\tau}(s)\right) d W(s) \\
& =\Gamma\left(f_{\tau}, g_{\tau}\right)(t) \text { for all } t \in \mathbb{R} .
\end{aligned}
$$

Thus $\Gamma(f, g)_{\tau}=\Gamma\left(f_{\tau}, g_{\tau}\right)$ which implies $\left\{\Gamma(f, g)_{\delta}, \delta \in \mathbb{R}\right\}$ is relatively compact in $S B C\left(\mathbb{R}, L^{2}(P, H)\right)$. Since $\Gamma$ is continuous from $S B C\left(\mathbb{R}, L^{2}(P, H)\right)$ into $S B C\left(\mathbb{R}, L^{2}(P, H)\right)$ then $\Gamma(f, g) \in S A P\left(\mathbb{R}, L^{2}(P, H)\right)$.

Theorem 4.21. Assume that $\left(\boldsymbol{H}_{3}\right)$ and $\left(\boldsymbol{H}_{5}\right)$ holds. Let $f, g \in \mathcal{E}\left(\mathbb{R}, L^{2}(P, H), \mu, \nu, r\right)$ then $\Gamma(f, g) \in \mathcal{E}\left(\mathbb{R}, L^{2}(P, H), \mu, \nu, r\right)$.

Proof. We have,

$$
\begin{aligned}
\Gamma(f, g)(t)= & \lim _{\lambda \rightarrow+\infty} \int_{-\infty}^{t} \mathcal{U}^{s}(t-s) \Pi^{s}\left(\widetilde{B}_{\lambda} X_{0} f(s)\right) d s \\
& +\lim _{\lambda \rightarrow+\infty} \int_{+\infty}^{t} \mathcal{U}^{u}(t-s) \Pi^{u}\left(\widetilde{B}_{\lambda} X_{0} f(s)\right) d s \\
& +\lim _{\lambda \rightarrow+\infty} \int_{-\infty}^{t} \mathcal{U}^{s}(t-s) \Pi^{s}\left(\widetilde{B}_{\lambda} X_{0} g(s)\right) d W(s) \\
& +\lim _{\lambda \rightarrow+\infty} \int_{+\infty}^{t} \mathcal{U}^{u}(t-s) \Pi^{u}\left(\widetilde{B}_{\lambda} X_{0} g(s)\right) d W(s)
\end{aligned}
$$




$$
\begin{aligned}
& \mathbb{E}\|\Gamma(f, g)(\theta)\|^{2}=\mathbb{E} \| \lim _{\lambda \rightarrow+\infty} \int_{-\infty}^{\theta} \mathcal{U}^{s}(t-s) \Pi^{s}\left(\widetilde{B}_{\lambda} X_{0} f(s)\right) d s \\
& +\lim _{\lambda \rightarrow+\infty} \int_{+\infty}^{\theta} \mathcal{U}^{u}(t-s) \Pi^{u}\left(\widetilde{B}_{\lambda} X_{0} f(s)\right) d s \\
& +\lim _{\lambda \rightarrow+\infty} \int_{-\infty}^{\theta} \mathcal{U}^{s}(t-s) \Pi^{s}\left(\widetilde{B}_{\lambda} X_{0} g(s)\right) d W(s) \\
& +\lim _{\lambda \rightarrow+\infty} \int_{+\infty}^{\theta} \mathcal{U}^{u}(t-s) \Pi^{u}\left(\widetilde{B}_{\lambda} X_{0} g(s)\right) d W(s) \|^{2} . \\
& \int_{-\tau}^{\tau} \sup _{\theta \in[t-r, t]} \mathbb{E}\|\Gamma(f, g)(\theta)\|^{2} d \mu(t) \leq \\
& \int_{-\tau}^{\tau} \sup _{\theta \in[t-r, t]} 4 \mathbb{E}\left(\widetilde{M}^{2} \bar{M}^{2} \int_{-\infty}^{\theta} e^{-2 \omega(t-s)}\left|\Pi^{s}\right|^{2}\|f(s)\|^{2} d s\right. \\
& +\widetilde{M}^{2} \bar{M}^{2} \int_{\theta}^{+\infty} e^{2 \omega(t-s)}\left|\Pi^{u}\right|^{2}\|f(s)\|^{2} d s+\widetilde{M}^{2} \bar{M}^{2} \int_{-\infty}^{\theta} e^{-2 \omega(t-s)}\left|\Pi^{s}\right|^{2}\|g(s)\|^{2} d s \\
& \left.+\widetilde{M^{2}} \bar{M}^{2} \int_{\theta}^{+\infty} e^{2 \omega(t-s)}\left|\Pi^{u}\right|^{2}\|g(s)\|^{2} d s\right) d \mu(t) \\
& \leq 4 \widetilde{M}^{2} \bar{M}^{2}\left[\int_{-\tau}^{\tau} \sup _{\theta \in[t-r, t]}\left(\int_{-\infty}^{\theta} e^{-2 \omega(t-s)}\left|\Pi^{s}\right|^{2} \mathbb{E}\|f(s)\|^{2} d s\right) d \mu(t)\right. \\
& +\int_{-\tau}^{\tau} \sup _{\theta \in[t-r, t]}\left(\int_{\theta}^{+\infty} e^{2 \omega(t-s)}\left|\Pi^{u}\right|^{2} \mathbb{E}\|f(s)\|^{2} d s\right) d \mu(t) \\
& \left.+\int_{-\tau}^{\tau} \sup _{\theta \in[t-r, t]}\left(\int_{-\infty}^{\theta} e^{-2 \omega(t-s)}\left|\Pi^{s}\right|^{2} \mathbb{E}\|g(s)\|^{2} d s+\int_{\theta}^{+\infty} e^{2 \omega(t-s)}\left|\Pi^{u}\right|^{2} \mathbb{E}\|g(s)\|^{2} d s\right) d \mu(t)\right] \\
& \leq 4 \widetilde{M}^{2} \bar{M}^{2}\left[\left|\Pi^{s}\right|^{2} \int_{-\tau}^{\tau} \sup _{\theta \in[t-r, t]}\left(\int_{-\infty}^{\theta} e^{-2 \omega(t-s)}\left(\mathbb{E}\|f(s)\|^{2}+\mathbb{E}\|g(s)\|^{2}\right) d s\right) d \mu(t)\right. \\
& \left.+\left|\Pi^{u}\right|^{2} \int_{-\tau}^{\tau} \sup _{\theta \in[t-r, t]}\left(\int_{\theta}^{+\infty} e^{2 \omega(t-s)}\left(\mathbb{E}\|f(s)\|^{2}+\mathbb{E}\|g(s)\|^{2}\right) d s\right) d \mu(t)\right]
\end{aligned}
$$

one the one hand using Fubini's theorem, we have

$$
\begin{array}{cc}
\left|\Pi^{s}\right|^{2} \int_{-\tau}^{\tau}\left[\sup _{\theta \in[t-r, t]} \int_{-\infty}^{\theta} e^{-2 \omega(t-s)}\left(\mathbb{E}\|f(s)\|^{2}+\mathbb{E}\|g(s)\|^{2}\right) d s\right] d \mu(t) \\
\leq \quad e^{\omega r}\left|\Pi^{s}\right|^{2} \int_{-\tau}^{\tau} \sup _{\theta \in[t-r, t]}\left(\int_{-\infty}^{\theta} e^{-2 \omega(t-s)}\left(\mathbb{E}\|f(s)\|^{2}+\mathbb{E}\|g(s)\|^{2}\right) d s\right) d \mu(t) \\
\leq \quad e^{\omega r}\left|\Pi^{s}\right|^{2} \int_{-\tau}^{\tau} \sup _{\theta \in[t-r, t]}\left(\int_{-\infty}^{t} e^{-2 \omega(t-s)}\left(\mathbb{E}\|f(s)\|^{2}+\mathbb{E}\|g(s)\|^{2}\right) d s\right) d \mu(t) \\
\leq \quad e^{\omega r}\left|\Pi^{s}\right|^{2} \int_{-\tau}^{\tau}\left(\int_{-\infty}^{t} e^{-2 \omega(t-s)}\left(\mathbb{E}\|f(s)\|^{2}+\mathbb{E}\|g(s)\|^{2}\right) d s\right) d \mu(t) \\
\leq \quad e^{\omega r}\left|\Pi^{s}\right|^{2} \int_{-\tau}^{\tau}\left(\int_{0}^{+\infty} e^{-2 \omega s}\left(\mathbb{E}\|f(t-s)\|^{2}+\mathbb{E}\|g(t-s)\|^{2}\right) d s\right) d \mu(t) \\
\leq \quad e^{\omega r}\left|\Pi^{s}\right|^{2} \int_{0}^{+\infty} e^{-2 \omega s} \int_{-\tau}^{\tau}\left(\mathbb{E}\|f(t-s)\|^{2}+\mathbb{E}\|g(t-s)\|^{2}\right) d \mu(t) d s
\end{array}
$$


By using Proposition(3.13) we deduce that

$$
\lim _{\tau \rightarrow+\infty} \frac{e^{-2 \omega s}}{\nu([-\tau, \tau])} \int_{-\tau}^{\tau}\left(\mathbb{E}\|f(t-s)\|^{2}+\mathbb{E}\|g(t-s)\|^{2}\right) d \mu(t) \rightarrow 0
$$

for all $s \in \mathbb{R}^{+}$and

$$
\frac{e^{-2 \omega s}}{\nu([-\tau, \tau])} \int_{-\tau}^{\tau}\left(\mathbb{E}\|f(t-s)\|^{2}+\mathbb{E}\|g(t-s)\|^{2}\right) d \mu(t) \leq \frac{e^{-2 \omega s} \mu([-\tau, \tau])}{\nu([-\tau, \tau])}\left(\|f\|_{\infty}^{2}+\|g\|_{\infty}^{2}\right)
$$

Since $f$ and $g$ are bounded functions, then the function $s \mapsto \frac{e^{-2 \omega s} \mu([-\tau, \tau])}{\nu([-\tau, \tau])}\left(\|f\|_{\infty}^{2}+\|g\|_{\infty}^{2}\right)$ belongs to $L^{1}([0,+\infty[)$ in view of the Lebesgue dominated convergence Theorem, it follows that $e^{\omega r} \lim _{\tau \rightarrow+\infty} \int_{0}^{+\infty} \frac{e^{-2 \omega s}}{\nu([-\tau, \tau])} \int_{-\tau}^{\tau}\left(\mathbb{E}\|f(t-s)\|^{2}+\mathbb{E}\|g(t-s)\|^{2}\right) d \mu(t) d s \rightarrow 0$.

On the other hand by Fubini's theorem, we also have

$$
\begin{aligned}
\left|\Pi^{u}\right|^{2} \int_{-\tau}^{\tau} & \sup _{\theta \in[t-r, t]}\left(\int_{\theta}^{+\infty} e^{2 \omega(t-s)}\left(\mathbb{E}\|f(s)\|^{2}+\mathbb{E}\|g(s)\|^{2}\right) d s\right) d \mu(t) \\
\leq & \left|\Pi^{u}\right|^{2} \int_{-\tau}^{\tau} \sup _{\theta \in[t-r, t]}\left(\int_{t-r}^{+\infty} e^{2 \omega(t-s)}\left(\mathbb{E}\|f(s)\|^{2}+\mathbb{E}\|g(s)\|^{2}\right) d s\right) d \mu(t) \\
\leq & \left|\Pi^{u}\right|^{2} \int_{-\tau}^{\tau}\left(\int_{t-r}^{+\infty} e^{2 \omega(t-s)}\left(\mathbb{E}\|f(s)\|^{2}+\mathbb{E}\|g(s)\|^{2}\right) d s\right) d \mu(t) \\
\leq & \left|\Pi^{u}\right|^{2} \int_{-\tau}^{\tau}\left(\int_{-\infty}^{r} e^{2 \omega s}\left(\mathbb{E}\|f(s)\|^{2}+\mathbb{E}\|g(s)\|^{2}\right) d s\right) d \mu(t) \\
\leq & \left|\Pi^{u}\right|^{2} \int_{-\infty}^{r}\left(\int_{-\tau}^{\tau} e^{2 \omega s}\left(\mathbb{E}\|f(s)\|^{2}+\mathbb{E}\|g(s)\|^{2}\right) d \mu(t)\right) d s
\end{aligned}
$$

Since the function $s \mapsto \frac{e^{2 \omega s}}{\nu([-\tau, \tau])}\left(\|f\|_{\infty}^{2}+\|g\|_{\infty}^{2}\right)$ belongs to $\left.\left.L^{1}(]-\infty, r\right]\right)$ resoning like above, it follows that

$\lim _{\tau \rightarrow+\infty} \int_{-\infty}^{\tau} e^{\omega s} \times \frac{1}{\nu([-\tau, \tau])}\left(\int_{-\tau}^{\tau} e^{2 \omega s}\left(\mathbb{E}\|f(s)\|^{2}+\mathbb{E}\|g(s)\|^{2}\right) d \mu(t)\right) d s=0$

Consequently

$$
\lim _{\tau \rightarrow+\infty} \frac{1}{\nu([-\tau, \tau])} \int_{-\tau}^{\tau} \sup _{\theta \in[t-r, t]} \mathbb{E}\|\Gamma(f, g)(\theta)\|^{2} d \mu(t)=0
$$

Thus, we obtain the desired result.

Theorem 4.22. Assume $\left(\boldsymbol{H}_{\boldsymbol{0}}\right),\left(\boldsymbol{H}_{\mathbf{1}}\right),\left(\boldsymbol{H}_{3}\right)$ and $\left(\boldsymbol{H}_{\boldsymbol{5}}\right)$ hold. Then equation (1.1) has a unique square mean $\mathrm{cl}(\mu, \nu)$-pseudo almost periodic solution of class $r$.

Proof. Since $f$ and $g$ are square mean $(\mu, \nu)$-pseudo almost periodic function, $f, g$ has a decomposition $f=f_{1}+f_{2}$ and $g=g_{1}+g_{2}$ where $f_{1}, g_{1} \in S A P\left(\mathbb{R} ; L^{2}(P, H)\right)$ and $f_{2}$, $g_{2} \in \mathcal{E}\left(\mathbb{R} ; L^{2}(P, H), \mu, \nu, r\right)$. Using Theorem(4.20), Theorem(4.2) and Theorem(4.17), we get the desired result.

Our next objective is to show the existence of square mean $(\mu, \nu)$-pseudo almost periodic solutions of class $r$ for the following problem

$$
d u(t)=\left[A u(t)+L\left(u_{t}\right)+f\left(t, u_{t}\right)\right] d t+g\left(t, u_{t}\right) d W(t) \text { for } t \in \mathbb{R}
$$


where $f: \mathbb{R} \times C \rightarrow L^{2}(P, H)$ and $g: \mathbb{R} \times C \rightarrow L^{2}(P, H)$ are two stochastic continuous processes. For the sequel, we formulate the following assumptions

$\left(\mathbf{H}_{\mathbf{6}}\right)$ Let $\mu, \nu \in \mathcal{M}$ and $f: \mathbb{R} \times C\left([-r, 0], L^{2}(P, H)\right) \rightarrow L^{2}(P, H)$ square mean $c l(\mu, \nu)$-pseudo almost periodic of class $r$ such that there exists a constant $L_{f}$ such that $\mathbb{E} \| f\left(t, \phi_{1}\right)-$ $f\left(t, \phi_{2}\right)\left\|^{2} \leq L_{f} \times \mathbb{E}\right\| \phi_{1}-\phi_{2} \|^{2}$ for all $t \in \mathbb{R}$ and $\phi_{1}, \phi_{2} \in C\left([-r, 0], L^{2}(P, H)\right)$.

$\left(\mathbf{H}_{\mathbf{7}}\right)$ Let $\mu, \nu \in \mathcal{M}$ and $g: \mathbb{R} \times C\left([-r, 0], L^{2}(P, H)\right) \rightarrow L^{2}(P, H)$ square mean $c l(\mu, \nu)$-pseudo almost periodic of class $r$ such that there exists a constant $L_{g}$ such that $\mathbb{E}\left\|g\left(t, \phi_{1}\right)-g\left(t, \phi_{2}\right)\right\|^{2} \leq L_{g} \times \mathbb{E}\left\|\phi_{1}-\phi_{2}\right\|^{2}$ for all $t \in \mathbb{R}$ and $\phi_{1}, \phi_{2} \in C\left([-r, 0], L^{2}(P, H)\right)$.

Theorem 4.23. Assume $\left(\boldsymbol{H}_{\boldsymbol{0}}\right),\left(\boldsymbol{H}_{\mathbf{1}}\right),\left(\boldsymbol{H}_{2}\right),\left(\boldsymbol{H}_{4}\right),\left(\boldsymbol{H}_{\boldsymbol{6}}\right)$ and $\left(\boldsymbol{H}_{\boldsymbol{7}}\right)$ hold. If

$$
\widetilde{M}^{2} \bar{M}^{2} \sup _{t \in \mathbb{R}}\left(\left|\Pi^{s}\right|^{2} \int_{-\infty}^{t} e^{-2 \omega(t-s)}\left(L_{f}^{2}+L_{g}^{2}\right) d s+\left|\Pi^{u}\right|^{2} \int_{t}^{+\infty} e^{2 \omega(t-s)}\left(L_{f}^{2}+L_{g}^{2}\right) d s\right)<\frac{1}{4}
$$

then equation (4.2) has a unique square mean $c l(\mu, \nu)$-pseudo almost periodic solution of class $r$.

Proof. Let $x$ be a function in $\operatorname{SPAP}\left(\mathbb{R}, L^{2}(P, H), \mu, \nu, r\right)$ from Theorem(4.11) the function $t \rightarrow x_{t}$ belongs to $S P A P\left(C\left([-r, 0] ; L^{2}(P, H), \mu, \nu, r\right)\right.$. Hence Theorem(4.16) implies that the function $g():.=f(., x$. $)$ is in $S P A P\left(\mathbb{R} ; L^{2}(P, H), \mu, \nu, r\right)$. Consider the following mapping:

$\mathcal{H}: S P A P\left(\mathbb{R} ; L^{2}(P, H), \mu, \nu, r\right) \rightarrow S P A P\left(\mathbb{R} ; L^{2}(P, H), \mu, \nu, r\right)$ defined for $t \in \mathbb{R}$ by

$$
\begin{aligned}
(\mathcal{H} x)(t)= & \lim _{\lambda \rightarrow+\infty} \int_{-\infty}^{t} \mathcal{U}^{s}(t-s) \Pi^{s} \widetilde{B}_{\lambda}\left(X_{0} f\left(s, x_{s}\right)\right) d s \\
& +\lim _{\lambda \rightarrow+\infty} \int_{+\infty}^{t} \mathcal{U}^{u}(t-s) \Pi^{u} \widetilde{B}_{\lambda}\left(X_{0} f\left(s, x_{s}\right)\right) d s \\
& +\lim _{\lambda \rightarrow+\infty} \int_{-\infty}^{t} \mathcal{U}^{s}(t-s) \Pi^{s} \widetilde{B}_{\lambda}\left(X_{0} g\left(s, x_{s}\right)\right) d W(s) \\
& +\lim _{\lambda \rightarrow+\infty} \int_{+\infty}^{t} \mathcal{U}^{u}(t-s) \Pi^{u} \widetilde{B}_{\lambda}\left(X_{0} g\left(s, x_{s}\right)\right) d W(s)
\end{aligned}
$$

From Theorem(4.20), Theorem(4.22) and Theorem(4.17), it suffices now to show that the operator $\mathcal{H}$ has a unique fixed point in $S P A P\left(\mathbb{R} ; L^{2}(P, H), \mu, \nu, r\right)$. Let $x_{1}, x_{2} \in$ $S P A P\left(\mathbb{R} ; L^{2}(P, H), \mu, \nu, r\right)$. Then we have

$$
\begin{aligned}
\mathbb{E}\left\|\mathcal{H} x_{1}(t)-\mathcal{H} x_{2}(t)\right\|^{2} \leq & 4 \mathbb{E}\left(\lim _{\lambda \rightarrow+\infty} \int_{-\infty}^{t} \| \mathcal{U}^{s}(t-s) \Pi^{s} \widetilde{B}_{\lambda}\left(X_{0}\left(f\left(s, x_{1 s}\right)-f\left(s, x_{2 s}\right)\right) d s \|^{2}\right)\right. \\
& +4 \mathbb{E}\left(\lim _{\lambda \rightarrow+\infty} \int_{t}^{+\infty} \| \mathcal{U}^{u}(t-s) \Pi^{u} \widetilde{B}_{\lambda}\left(X_{0}\left(f\left(s, x_{2 s}\right)-f\left(s, x_{1 s}\right)\right) d s \|^{2}\right)\right. \\
& +4 \mathbb{E}\left(\lim _{\lambda \rightarrow+\infty} \int_{-\infty}^{t} \| \mathcal{U}^{s}(t-s) \Pi^{s} \widetilde{B}_{\lambda}\left(X_{0}\left(g\left(s, x_{1 s}\right)-g\left(s, x_{2 s}\right)\right) d s \|^{2}\right)\right. \\
& +4 \mathbb{E}\left(\lim _{\lambda \rightarrow+\infty} \int_{t}^{+\infty} \| \mathcal{U}^{u}(t-s) \Pi^{u} \widetilde{B}_{\lambda}\left(X_{0}\left(g\left(s, x_{2 s}\right)-g\left(s, x_{1 s}\right)\right) d s \|^{2}\right)\right.
\end{aligned}
$$




$$
\begin{aligned}
\leq & 4 \widetilde{M}^{2} \bar{M}^{2} \mathbb{E}\left(\left|\Pi^{s}\right|^{2} \int_{-\infty}^{t} e^{-2 \omega(t-s)}\left(L_{f}^{2}+L_{g}^{2}\right)\left\|x_{1 s}-x_{2 s}\right\|^{2} d s\right. \\
& \left.+\left|\Pi^{u}\right|^{2} \int_{t}^{+\infty} e^{2 \omega(t-s)}\left(L_{f}^{2}+L_{g}^{2}\right)\left\|x_{1 s}-x_{2 s}\right\|^{2} d s\right) \\
\leq & 4 \widetilde{M}^{2} \bar{M}^{2} \mathbb{E}\left(\left\|x_{1}-x_{2}\right\|^{2}\right) \sup _{t \in \mathbb{R}}\left(\left|\Pi^{s}\right|^{2} \int_{-\infty}^{t} e^{-2 \omega(t-s)}\left(L_{f}^{2}+L_{g}^{2}\right) d s\right. \\
& \left.+\left|\Pi^{u}\right|^{2} \int_{t}^{+\infty} e^{2 \omega(t-s)}\left(L_{f}^{2}+L_{g}^{2}\right) d s\right) .
\end{aligned}
$$

This means that $\mathcal{H}$ is a strict contraction. Thus by Banach? fixed point theorem, $\mathcal{H}$ has a unique fixed point $u$ in $S P A P\left(\mathbb{R} ; L^{2}(P, H), \mu, \nu, r\right)$. We conclude that equation (4.2), has one and only one square mean $c l(\mu, \nu)$-pseudo almost periodic solution of class $r$.

Proposition 4.24. Assume $\left(\boldsymbol{H}_{\boldsymbol{O}}\right),\left(\boldsymbol{H}_{\mathbf{1}}\right),\left(\boldsymbol{H}_{2}\right),\left(\boldsymbol{H}_{4}\right)$ and $f, g$ are lipschitz continuous with respect the second argument. If

$$
\operatorname{Lip}(f)=\operatorname{Lip}(g)<\left(\frac{\omega}{4 \bar{M}^{2} \widetilde{M}^{2}\left(\left|\Pi^{s}\right|^{2}+\left|\Pi^{u}\right|^{2}\right)}\right)^{\frac{1}{2}}
$$

then equation (5.1) has a unique cl $(\mu, \nu)$-pseudo almost periodic solution of class $r$, where $\operatorname{Lip}(f)$, Lip $(g)$ are respectively the Lipschitz constant of $f$ and $g$.

Proof. Let us pose $k=\operatorname{Lip}(f)=\operatorname{Lip}(g)$, we have

$$
\begin{aligned}
\mathbb{E}\left\|\mathcal{H} x_{1}(t)-\mathcal{H} x_{2}(t)\right\|^{2} \leq & 4 \widetilde{M}^{2} \bar{M}^{2} \mathbb{E}\left(\left\|x_{1}-x_{2}\right\|^{2}\right) \sup _{t \in \mathbb{R}}\left(\left|\Pi^{s}\right|^{2} \int_{-\infty}^{t} e^{-2 \omega(t-s)}\left(L_{f}^{2}+L_{g}^{2}\right) d s\right. \\
& \left.+\left|\Pi^{u}\right|^{2} \int_{t}^{+\infty} e^{2 \omega(t-s)}\left(L_{f}^{2}+L_{g}^{2}\right) d s\right) \\
\leq & 4 \widetilde{M}^{2} \bar{M}^{2} \mathbb{E}\left(\left\|x_{1}-x_{2}\right\|^{2}\right) \sup _{t \in \mathbb{R}}\left(\left|\Pi^{s}\right|^{2} \int_{-\infty}^{t} 2 k^{2} e^{-2 \omega(t-s)} d s+\left|\Pi^{u}\right|^{2} \int_{t}^{+\infty} 2 k^{2} e^{2 \omega(t-s)} d s\right) \\
\leq & \frac{4 k^{2} \widetilde{M}^{2} \bar{M}^{2}\left(\left|\Pi^{s}\right|^{2}+\left|\Pi^{u}\right|^{2}\right)}{\omega} \mathbb{E}\left(\left\|x_{1}-x_{2}\right\|^{2}\right) .
\end{aligned}
$$

Consequently $\mathcal{H}$ is a strict contraction if $k^{2}<\frac{\omega}{4 \widetilde{M}^{2} \bar{M}^{2}\left(\left|\Pi^{s}\right|^{2}+\left|\Pi^{s}\right|^{2}\right)}$.

\section{ApplicATiON}

For illustration, we propose to study the existence of solutions for the following model

$$
\left\{\begin{aligned}
d z(t, x)= & \frac{\partial^{2}}{\partial x^{2}} z(t, x) d t+\left[\int_{-r}^{0} G(\theta) z(t+\theta, x) d \theta+\sin (t)+\sin (\sqrt{2} t)+\arctan (t)\right. \\
& +\int_{-r}^{0} h(\theta, z(t+\theta, x) d \theta] d t+\left[\frac{\cos (t)}{2+\cos (\sqrt{2} t)}+\arctan (t)\right. \\
& \left.+\int_{-r}^{\theta} h(\theta, z(t+\theta, x)) d \theta\right] d W(t) \\
z(t, 0)= & z(t, \pi)=0 \text { for } t \in \mathbb{R}
\end{aligned}\right.
$$

Where $G:[-r, 0] \rightarrow \mathbb{R}$ is a continuous function and $h:[-r, 0] \times \mathbb{R} \rightarrow \mathbb{R}$ is continuous, Lipschitzian with respect to the second argument. $W(t)$ is a two-sided and standard onedimensional Brownian notion defined on the filtered probability space $\left(\Omega, \mathcal{F}, P, \mathcal{F}_{t}\right)$ with 
$\mathcal{F}_{t}=\sigma\{W(u)-W(v) \mid u, v \leq t\}$. To rewrite equation (5.1) in the abstract form, we introduce the space $H=L^{2}((0, \pi))$. Let $A: D(A) \rightarrow L^{2}((0, \pi))$ defined by

$$
\left\{\begin{array}{l}
D(A)=\mathbf{H}^{1}((0, \pi)) \cap \mathbf{H}_{0}^{1}((0,1)) \\
A y(t)=y^{\prime \prime}(t) \text { for } t \in(0, \pi) \text { and } y \in D(A)
\end{array}\right.
$$

Then $A$ generates a $C_{0}$-semigroup $(\mathcal{U}(t))_{t \geq 0}$ on $L^{2}((0, \pi))$ given by

$$
(\mathcal{U}(t) x)(r)=\sum_{n=1}^{\infty} e^{-n^{2} \pi^{2} t}<x, e_{n}>_{L^{2}} e_{n}(r)
$$

Where $e_{n}(r)=\sqrt{2} \sin (n \pi r)$ for $n=1,2, \ldots$, and $\|\mathcal{U}(t)\| \leq e^{-\pi^{2} t}$ for all $t \geq 0$. Thus $\bar{M}=1$ and $\omega=\pi^{2}$. Then $A$ satisfied the Hille-Yosida condition in $L^{2}((0, \pi))$. Moreover the part $A_{0}$ of $A$ in $\overline{D(A)}$. It follows that $\left(\mathbf{H}_{\mathbf{0}}\right)$ and $\left(\mathbf{H}_{\mathbf{1}}\right)$ are satisfied.

We define $f: \mathbb{R} \times C \rightarrow L^{2}((0, \pi))$ and $L: C \rightarrow L^{2}((0, \pi))$ as follows

$$
\begin{aligned}
f(t, \phi)(x) & =(\sin (t)+\sin (\sqrt{2} t))+\arctan (t)+\int_{-r}^{\theta} h(\theta, \phi(\theta)(x)) d \theta \\
g(t, \phi)(x) & =\frac{\cos (t)}{2+\cos (\sqrt{2} t)}+\arctan (t)+\int_{-r}^{\theta} h(\theta, \phi(\theta)(x)) d \theta \\
L(\phi)(x) & =\int_{-r}^{\theta} G(\theta, \phi(\theta)(x)) d \theta \text { for }-r \leq \theta \leq 0 \text { and } x \in(0, \pi)
\end{aligned}
$$

let us pose $v(t)=z(t, x)$. Then equation(5.1) takes the following abstract form

$$
d v(t)=\left[A v(t)+L\left(v_{t}\right)+f\left(t, v_{t}\right)\right] d t+g\left(t, v_{t}\right) d W(t) \text { for } t \in \mathbb{R}
$$

Consider the measures $\mu$ and $\nu$ where its Radon-Nikodyn derivative are respectively $\rho_{1}, \rho_{2}$ : $\mathbb{R} \rightarrow \mathbb{R}$ defined by

$$
\rho_{1}(t)=\left\{\begin{array}{r}
1 \text { for } t>0 \\
e^{t} \text { for } t \leq 0
\end{array}\right.
$$

and

$$
\rho_{2}(t)=|t| \text { for } t \in \mathbb{R}
$$

i.e $d \mu(t)=\rho_{1}(t) d t$ and $d \nu(t)=\rho_{2}(t) d t$ where $d t$ denotes the Lebesgue measure on $\mathbb{R}$ and

$$
\mu(A)=\int_{A} \rho_{1}(t) d t \text { for } \nu(A)=\int_{A} \rho_{2}(t) d t \text { for } A \in \mathcal{B} .
$$

From [6] $\mu, \nu \in \mathcal{M}, \mu, \nu$ satisfy $\left(\mathbf{H}_{\mathbf{4}}\right)$ and $\sin (t)+\sin (\sqrt{2} t)+\frac{\pi}{2}$ is almost periodic.

We have

$$
\limsup _{\tau \rightarrow+\infty} \frac{\mu([-\tau, \tau])}{\nu([-\tau, \tau])}=\limsup _{\tau \rightarrow+\infty} \frac{\int_{-\tau}^{0} e^{t} d t+\int_{0}^{\tau} d t}{2 \int_{-\tau}^{0} t d t}=\limsup _{\tau \rightarrow+\infty} \frac{1-e^{-\tau}+\tau}{\tau^{2}}=0<\infty
$$

which implies that $\left(\mathbf{H}_{\mathbf{2}}\right)$ is satisfied.

For all $t \in \mathbb{R}, \frac{\pi}{2} \leq \arctan t \leq \frac{\pi}{2}$ therefore, for all $\theta \in[t-r, t], \arctan (t-r) \leq \arctan (\theta)$. It follows $\left|\arctan \theta-\frac{\pi}{2}\right|=\frac{\pi}{2}-\arctan \theta \leq\left|\arctan (t-r)-\frac{\pi}{2}\right|=\frac{\pi}{2}-\arctan (t-r)$, implies that 
$\left|\arctan \theta-\frac{\pi}{2}\right|^{2} \leq\left|\arctan (t-r)-\frac{\pi}{2}\right|^{2}$ hence $\sup _{\theta \in[t-r, t]} \mathbb{E}\left|\arctan \theta-\frac{\pi}{2}\right|^{2} \leq \mathbb{E}\left|\arctan (t-r)-\frac{\pi}{2}\right|^{2}$.

One the one hand, we have the following:

$$
\begin{aligned}
\frac{1}{\nu([-\tau, \tau])} \int_{0}^{\tau} \mathbb{E}\left|\arctan (t-r)-\frac{\pi}{2}\right|^{2} d t & =\frac{1}{\nu([-\tau, \tau])} \int_{0}^{\tau} \mathbb{E}\left(\frac{\pi}{2}-\arctan (t-r)\right)^{2} d t \\
& \leq \frac{1}{\nu([-\tau, \tau])} \int_{0}^{\tau} \frac{\pi^{2}}{4} d t \\
& \leq \frac{\pi^{2}}{4 \tau} \rightarrow 0 \text { as } \rightarrow+\infty
\end{aligned}
$$

On the other hand we have

$$
\begin{aligned}
\frac{1}{\nu([-\tau, \tau])} \int_{-\tau}^{0} \mathbb{E}\left|\arctan (t-r)-\frac{\pi}{2}\right|^{2} e^{t} d t & \leq \frac{1}{\nu([-\tau, \tau])} \int_{0}^{\tau} \frac{\pi^{2}}{4} e^{t} d t \\
& \leq \frac{\pi^{2}\left(1-e^{-\tau}\right)}{4 \tau} \rightarrow 0 \text { as } \rightarrow+\infty
\end{aligned}
$$

Consequently

$$
\lim _{\tau \rightarrow+\infty} \frac{1}{\nu([-\tau, \tau])} \int_{-\tau}^{+\tau} \sup _{\theta \in[t-r, t]} \mathbb{E}\left|\arctan \theta-\frac{\pi}{2}\right|^{2} d \mu(t)=0
$$

It follows that $t \mapsto \arctan t-\frac{\pi}{2}$ is square mean $(\mu, \nu)$-ergodic of class $r$, consequently, $f$ is uniformly square mean $(\mu, \nu)$-pseudo almost periodic of class $r$. Moreover, $L$ is bounded linear operator from $C$ to $L^{2}\left(P, L^{2}((0, \pi))\right.$.

Let $k$ be the lipschiz constant of $h$, then for every $\phi_{1}, \phi_{2} \in C$ and $t \geq 0$, we have

$$
\begin{aligned}
\mathbb{E}\left\|f\left(t, \phi_{1}\right)(x)-f\left(t, \phi_{2}\right)(x)\right\|^{2} & =\mathbb{E}\left\|\int_{-r}^{0}\left[h\left(\theta, \phi_{1}(\theta)(x)\right)-h\left(\theta, \phi_{2}(\theta)(x)\right)\right]\right\| \|^{2} d \theta \\
& \leq \int_{-r}^{0} \mathbb{E}\left\|h\left(\theta, \phi_{1}(\theta)(x)\right)-h\left(\theta, \phi_{2}(\theta)(x)\right)\right\|^{2} d \theta \\
& \leq \int_{-r}^{0} k \mathbb{E}\left\|\phi_{1}(\theta)(x)-\phi_{2}(\theta)(x)\right\|^{2} d \theta \\
\mathbb{E}\left\|f\left(t, \phi_{1}\right)(x)-f\left(t, \phi_{2}\right)(x)\right\|^{2} & \leq k r \sup _{-r \leq \theta \leq 0} \mathbb{E}\left\|\phi_{1}(\theta)(x)-\phi_{2}(\theta)(x)\right\|^{2} \\
\leq & k r \alpha \mathbb{E}\left\|\phi_{1}(\theta)(x)-\phi_{2}(\theta)(x)\right\|^{2} \text { for a certain } \alpha \in \mathbb{R}_{+}
\end{aligned}
$$

Consequently, we conclude that $f$ and $g$ are Lipschitz continuous and $c l(\mu, \nu)$-pseudo almost periodic of class $\mathrm{r}$.

Moreover, since $h$ is stochastically bounded, i.e $\mathbb{E}\|h(t, \phi(t)(x))\| \leq \beta, t \in \mathbb{R}$, we have

$$
\begin{aligned}
\mathbb{E}\|g(t, \phi)(x)\|^{2} & \leq \frac{4+\pi}{2}+\int_{-r}^{0} \mathbb{E}\|h(\theta, \phi(\theta)(x))\|^{2} d \theta \\
& \leq \frac{4+\pi}{2}+r \cdot \beta \\
& \leq \beta_{1} \text { with } \beta_{1}=\frac{4+\pi}{2}+r . \beta .
\end{aligned}
$$


Which implies that $g$ satisfies $\left(\mathbf{H}_{\mathbf{5}}\right)$

For the hyberbolicity, we suppose that

$\left(\mathbf{H}_{\mathbf{8}}\right) \int_{-r}^{0}|G(\theta)| d \theta<1$.

Proposition 5.1. [11] Assume that $\left(\boldsymbol{H}_{\boldsymbol{6}}\right)$ and $\left(\boldsymbol{H}_{\boldsymbol{7}}\right)$ holds. Then the semigroup $(U(t))_{t \geq 0}$ is hyperbolic.

Then by Proposition (4.24) we deduce the following result.

Theorem 5.2. Under the above assumptions, if Lip $(h)$ is small enough, then equation (5.1) has a unique $\mathrm{cl}(\mu, \nu)$-pseudo almost periodic solution $\nu$ of class $r$.

\section{REFERENCES}

[1] M. Adimy, A. Elazzouzi, K. Ezzinbi, Reduction principle and dynamic behaviors for a class of partial functional differential equations, Nonlinear Anal., Theory Methods Appl. 71 (2009) 1709-1727. https: //doi.org/10.1016/j.na.2009.01.008.

[2] M. Adimy, A. Elazzouzi, K. Ezzinbi, Bohr-Neugebauer type theorem for some partial neutral functional differential equations, Nonlinear Anal., Theory Methods Appl. 66 (2007) 1145-1160. https://doi.org/ $10.1016 / j$.na.2006.01.011.

[3] M. Adimy, K. Ezzinbi, M. Laklach, Spectral decomposition for partial neutral functional differential equations, Canadian Appl. Math. Quart. 9 (1) (2001) 1-34.

[4] J. Blot, P. Cieutat, K. Ezzinbi, Measure theory and pseudo almost automorphic functions: New developments and applications, Nonlinear Anal., Theory Methods Appl. 75 (2012) 2426-2447. https: //doi.org/10.1016/j.na.2011.10.041.

[5] P. H. Bezandry, T. Diagana Square-mean almost periodic solutions nonautonomous stochastic differential equations. Electron. J. Differ. Equ. 2007 (2007) 117.

[6] J. Blot, P. Cieutat, K. Ezzinbi, New approach for weighted pseudo-almost periodic functions under the light of measure theory, basic results and applications, Appl. Anal. 92 (2013) 493-526. https://doi.org/ 10.1080/00036811.2011.628941.

[7] T. Diagana, K. Ezzinbi and M. Miraoui, Pseudo-almost periodic and pseudo-almost automorphic solutions to some evolution equations involving theoretical measure theory, CUBO 16 (02) (2014) 01-31.

[8] T. Diagana, Semilinear Evolution Equations and Their Applications, Springer, (2018).

[9] M.A. Diop, K. Ezzinbi, M.M. Mbaye, Measure theory and $S^{2}$-pseudo almost periodic and automorphic process: application to stochastic evolution equations, Afr. Mat. 26 (2015) 779-812. https://doi.org/ 10.1007/s13370-014-0247-x.

[10] M. Diop, K. Ezzinbi, M. Mbaye, Measure theory and square-mean pseudo almost periodic and automorphic process: application to stochastic evolution equations, Bull. Malays. Math. Sci. Soc. 41 (2018) 287-310.

[11] K. Ezzinbi, S. Fatajou, N'Guérékata, Pseudo almost automorphic solutions for dissipative differential equations in Banach spaces, J. Math. Anal. Appl. 351 (2009) 765-772. https://doi.org/10.1016/j.jmaa. 2008.11.017.

[12] K. Ezzinbi, S. Fatajou, G.M. N'Guérékata, $C^{n}$-almost automorphic solutions for partial neutral functional differential equations, Appl. Anal. 86 (9) (2007) 1127-1146. https://doi.org/10.1080/ 00036810701584591.

[13] W. Rudin, Real and Complex Analysis, 3rd ed. McGraw-Hill Book Compagny, New-York, 1986.

[14] I. Zabsonre, H. Toure, Pseudo-almost periodic and pseudo-almost automorphic solutions of class $r$ under the light of measure theory, Afr. Diaspora J. Math. 19 (1) (2016) 58-86. 